\title{
Fast automatic myocardial segmentation in 4D cine CMR datasets
}

\author{
Sandro Queirós ${ }^{\mathrm{a}, \mathrm{b}, *, 1}$, Daniel Barbosa ${ }^{\mathrm{a}, \mathrm{c}, \mathrm{d}, 1}$, Brecht Heyde ${ }^{\mathrm{a}}$, Pedro Morais ${ }^{\mathrm{a}, \mathrm{b}}$, João L. Vilaça ${ }^{\mathrm{b}, \mathrm{d}}$, \\ Denis Friboulet ${ }^{c}$, Olivier Bernard ${ }^{c}$, Jan D’hooge ${ }^{a}$ \\ ${ }^{a}$ Lab on Cardiovascular Imaging and Dynamics, KU Leuven, Belgium \\ ${ }^{\mathrm{b}}$ ICVS/3B's - PT Government Associate Laboratory, Braga/Guimarães, Portugal \\ ' Université de Lyon, CREATIS, CNRS UMR5220, INSERM U630, Université Lyon 1, INSA-Lyon, France \\ ${ }^{\mathrm{d}}$ DIGARC - Polytechnic Institute of Cávado and Ave (IPCA), Barcelos, Portugal
}

\section{A R T I C L E I N F O}

\section{Article history:}

Received 19 December 2013

Received in revised form 5 May 2014

Accepted 6 June 2014

Available online 19 June 2014

\section{Keywords:}

Fast image segmentation

Cardiac cine MRI

Left ventricle segmentation

Automatic initialization

\begin{abstract}
A B S T R A C T
A novel automatic 3D+time left ventricle (LV) segmentation framework is proposed for cardiac magnetic resonance (CMR) datasets. The proposed framework consists of three conceptual blocks to delineate both endo and epicardial contours throughout the cardiac cycle: (1) an automatic 2D mid-ventricular initialization and segmentation; (2) an automatic stack initialization followed by a 3D segmentation at the enddiastolic phase; and (3) a tracking procedure. Hereto, we propose to adapt the recent B-spline Explicit Active Surfaces (BEAS) framework to the properties of CMR images by integrating dedicated energy terms. Moreover, we extend the coupled BEAS formalism towards its application in 3D MR data by adapting it to a cylindrical space suited to deal with the topology of the image data. Furthermore, a fast stack initialization method is presented for efficient initialization and to enforce consistent cylindrical topology. Finally, we make use of an anatomically constrained optical flow method for temporal tracking of the LV surface.

The proposed framework has been validated on 45 CMR datasets taken from the 2009 MICCAI LV segmentation challenge. Results show the robustness, efficiency and competitiveness of the proposed method both in terms of accuracy and computational load.
\end{abstract}

() 2014 Elsevier B.V. All rights reserved.

\section{Introduction}

Cardiovascular diseases (CVDs) are the leading cause of death in the world. According to the World Health Organization (2012), an estimated 17.3 million people died from CVDs in 2008, which represents $30 \%$ of all global deaths. This report and the known global population aging highlight the increasing need for powerful and efficient techniques for diagnosis and treatment follow-up of patients with CVDs. Among others, assessment of the left ventricular morphology and global function using non-invasive cardiac imaging is an interesting technique for such purpose.

Cardiac magnetic resonance (CMR) imaging is a successful and promising modality that has already demonstrated its excellent accuracy and reproducibility (Miller et al., 2012a). The quantification of left ventricular volumes, mass (LVM) and ejection fraction (EF) is crucial for cardiac diagnosis, requiring the delineation of

\footnotetext{
* Corresponding author at: ICVS/3B's - PT Government Associate Laboratory, Braga/Guimarães, Portugal. Tel.: +351 253604891.

E-mail addresses: sandroqueiros@ecsaude.uminho.pt (S. Queirós), dbarbosa@ ipca.pt (D. Barbosa).

${ }^{1}$ Joint first authorship.
}

endocardial and epicardial contours of the left ventricle (LV) from cine MR images. In clinical practice, manual segmentation is usually performed, being a tedious, time consuming and unpractical task (Petitjean and Dacher, 2011), normally requiring between 6 and 20 min to segment all slices covering the whole LV in end-diastolic (ED) and end-systolic (ES) cardiac phases only (Miller et al., 2012b; Hautvast et al., 2012; Petitjean and Dacher, 2011). Moreover, manual delineation is prone to intra- and inter-observer variability, mainly associated with the choice of ED and ES phases, the choice of the most basal LV slice and the chosen endocardial edge selection approach (Anderson et al., 2007; Miller et al., 2012b). In their recent study, Miller et al. (2012b) presented the "real-world" variability associated with manual contouring, showing that significant differences in the quantification of LV indices occur depending on the used analysis method. In practice, such differences would require specific reference ranges depending on the contouring methodology used by the physician.

To date, several methods have been presented for (semi-)automated LV CMR segmentation. According to the recent review in Petitjean and Dacher (2011), these methods can be divided in two main categories: weak or no prior methods and strong prior methods. The first uses weak assumptions, such as spatial or 
intensity-based relations and anatomical assumptions, and include image-based techniques (threshold, dynamic programming, etc.) (Hu et al., 2012; Liu et al., 2012), pixel classification methods (clustering, Gaussian mixture model fitting, etc.) (Ulén et al., 2013; Pednekar et al., 2006) and deformable models (active contours, level-sets and variants) (Chen et al., 2008; Pluempitiwiriyawej et al., 2005; Paragios, 2002; Lynch et al., 2008; Constantinides et al., 2012; Cordero-Grande et al., 2011; Ben Ayed et al., 2012). Strong prior methods include shape prior based deformable models, active shape and appearance models and atlas based methods, which focus on higher-level shape and intensity information and normally require a training dataset with manual segmentations (Ulén et al., 2013; Eslami et al., 2013). An extensive overview of the different available methodologies can be found in Petitjean and Dacher (2011).

Although several approaches are already automatic, optimal boundaries assessment from base to apex is still lacking, usually requiring the physician to manually correct the contours (Hautvast et al., 2012; Petitjean and Dacher, 2011). Therefore, the LV segmentation remains an open problem with significant clinical value, easily noticed by the constant attention in the research field (Cordero-Grande et al., 2011; Constantinides et al., 2012; Hu et al., 2012; Liu et al., 2012; Ben Ayed et al., 2012; Ulén et al., 2013; Eslami et al., 2013). In 2012, Hu et al. (2012) presented an image-based framework using a Gaussian-mixture model for endocardial delineation and region restricted dynamic programming for epicardial contouring. Similarly, Liu et al. (2012) also used region restricted dynamic programming for epicardial contouring, but opted for topological stable-state thresholding to delineate the endocardium. Both methodologies are fast, automatic and proven to be robust in a publicly available database. In the same year, Ben Ayed et al. (2012) presented a fast LV segmentation framework based on max-flow optimization of discrete cost functions encompassing global intensity and geometry constraints based on the Bhattacharyya similarity. Although sufficiently fast in segmenting all cardiac phases, it still required a manual initialization in the first frame. More recently, in 2013, Ulén et al. (2013) presented a multi-region segmentation framework based on graph cuts and Lagrangian duality. They evaluated it for the segmentation of cardiac structures in CMR, namely LV and right ventricle (RV) cavities, myocardium and trabeculae and papillary muscles (TPMs). Eslami et al. (2013) presented a framework based on guided random walks for LV segmentation with high-level prior knowledge but without the need for statistical shape models. Instead, they guide the segmentation using the closest subject in the database. Both Ulén et al. (2013) and Eslami et al. (2013) require some user interaction and most importantly the incorporation of training datasets, which are normally efficient but intrinsically link their ability to handle clinical variability to the amount of different morphological and physiological cardiac patterns included in the training set.

The effort seen in the research field reinforce the difficulties in designing a framework that could deal with the segmentation challenges presented in CMR images (Petitjean and Dacher, 2011; Cordero-Grande et al., 2011; Chen et al., 2008), such as:

- the poor contrast between myocardium and surrounding structures (e.g., lungs and liver);

- the heterogeneities in the LV cavity brightness due to blood flow;

- the presence of TPMs with similar signal intensities to the myocardium;

- the partial volume effects (PVE) in apical slices due to the limited MRI resolution;

- the inherent noise associated with cine CMR, which is caused by motion artifacts, heart dynamics and intensity inhomogeneity;
- and the considerable shape and intensity variability of the LV across patients, notably in pathological cases.

Thus, to be of interest in "real-world" clinical practice, a robust segmentation framework should be accurate, flexible and generic enough to be able to segment a multitude of LV shapes with various myocardial contraction patterns. Moreover, the time required to segment is a critical factor for success when introducing a methodology in daily clinical practice.

Among the multitude of methodologies, active contour-based methods are a flexible framework, allowing easy incorporation of prior knowledge about the object's shape and expected intensity distribution, as well as of temporal constraints (Petitjean and Dacher, 2011). In these methods, the shape to recover is captured by propagating an evolving interface while minimizing an energy functional that reflects the object's properties (Barbosa et al., 2012). As image feature, the energy terms are usually classified as either edge-based or region-based. Edge-based energy terms use image gradients to identify object boundaries and attract the contours toward edges (Paragios, 2002; Pluempitiwiriyawej et al., 2005; Chen et al., 2008), while region-based ones use regional properties inside and outside the object to evolve the contour (Paragios, 2002; Pluempitiwiriyawej et al., 2005; Chen et al., 2008; Lankton and Tannenbaum, 2008; Barbosa et al., 2010). Some authors combine both to improve accuracy, while adding smoothness constraints, contour interaction and/or shape priors (Paragios, 2002; Pluempitiwiriyawej et al., 2005; Chen et al., 2008; Ben Ayed et al., 2009). Moreover, temporal integration is sometimes included for 2D+time or 3D+time segmentation (Lynch et al., 2008; Ben Ayed et al., 2012; Hautvast et al., 2006). Note that active contours are usually dependent on their initialization, highlighting the importance of this preliminary step (Petitjean and Dacher, 2011). Thus, active contours usually require some user interaction, either in a minimal way (Paragios, 2002; Pluempitiwiriyawej et al., 2005; Chen et al., 2008) or in an advanced way (e.g., requiring a first manual contour) (Ben Ayed et al., 2009; Ben Ayed et al., 2012; Hautvast et al., 2006). Nevertheless, several authors proposed automatic initialization procedures, focusing on obtaining a robust initialization while avoiding the variability introduced by user input (Lu et al., 2009; Ciofolo et al., 2008; Queirós et al., 2013).

The focus of the present work was to develop an automatic 3D+time myocardial segmentation framework for CMR datasets with accurate shape delineation and low computational burden, to be of interest in daily clinical practice. To this end, we propose a novel framework corresponding to the 3D+time extension of the preliminary work previously presented in Queirós et al. (2013). This work encompassed a fast automatic initialization approach capable of detecting LV elliptical annular shapes and an adapted version of the B-spline Explicit Active Surfaces (BEAS) framework, initially proposed in Barbosa et al. (2012), to 2D CMR image segmentation (Queirós et al., 2013). In the present paper, the key novelties introduced are threefold:

- First, we extend the coupled BEAS framework introduced in Queirós et al. (2013) towards its application in 3D MR data. To this end, we adapt the mathematical formalism to a cylindrical space suited to deal with the topology of the image data. Furthermore, we provide a rigorous mathematical derivation to support the variational formulation of the coupled segmentation problem.

- Secondly, we develop a fast stack initialization method in order to provide an efficient and spatially consistent initialization for the 3D segmentation algorithm. This step also enables to model the 3D LV surface in a parametric cylindrical space, since it partially compensates for slice misalignment. This is achieved 


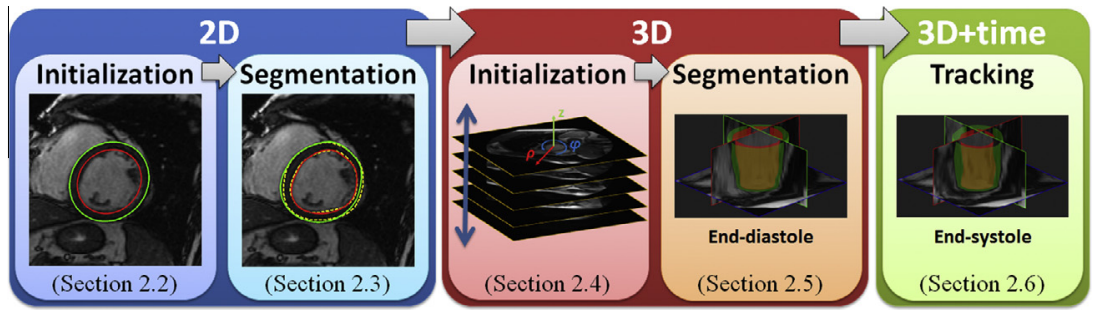

Fig. 1. General overview of the proposed fully automatic 3D+time LV segmentation framework for CMR datasets.

through sequential threshold-based segmentation of adjacent slices, using the information gathered from the automatic segmentation of the mid-ventricular short-axis (SAX) slice.

- Lastly, we introduce a hybrid energy term making use of both region and edge-based image features. Although such strategy has been previously applied to level-set frameworks (Lin et al., 2003; Paragios, 2002), it is the first time that edge-based terms are used in the BEAS formalism.

Additionally, the LV surface was tracked throughout the cardiac cycle making use of an anatomical affine optical flow method, initially presented for ultrasound (US) data (Barbosa et al., 2013b).

The accuracy of the proposed framework was assessed against manually extracted references for 45 publicly available SAX datasets from the 2009 MICCAI LV segmentation challenge, including both healthy subjects and patients. The results were compared to state-of-the-art methods that used the same database and a very competitive performance was achieved by our algorithm.

This paper is structured as follows. In Section 2, we focus on the detailed description of the proposed segmentation framework. In Section 3, we discuss the implementation details of our method, particularly the choice of parameters and their influence. Section 4 presents the validation experiments, whose results are presented in Section 5. In Section 6, we evaluate and discuss the performance of each module of our framework and compare with state-of-theart methods. The main conclusions of this paper are given in Section 7.

\section{Methodology}

\subsection{General overview}

The ultimate goal of the proposed approach is to obtain an accurate and fast 3D+time LV segmentation framework for CMR datasets in order to assess both cardiac morphology and global function. We thus propose to divide the approach in 3 conceptual blocks (Fig. 1).

The first block corresponds to an automatic 2D mid-ventricular segmentation in the ED phase, divided in two modules. The first module focuses on obtaining an initial contour for a mid-ventricular slice. Hereto, we use the fast and robust automatic initialization procedure presented in a previous contribution (Queirós et al., 2013). The second module uses the BEAS framework and a specifically designed energy functional for 2D segmentation of the midventricular slice (whose expression is given in Section 2.3).

The second block presents an extension to 3D segmentation of BEAS, with a hybrid region-based and edge-based epicardial energy functional. However, as it requires all slices in the ED phase to be initialized, an automatic stack initialization is first performed using a simple threshold-based BEAS formulation using information from the 2D segmentation result (i.e., first block). This step also allows to estimate possible slice misalignment to achieve spatial continuity during the 3D segmentation, as further explained in Sections 2.4 and 2.5.

At last, the third block focuses on tracking the segmented contours of the ED phase over all cardiac phases, ultimately obtaining both endo and epicardial contours in the ES phase. Hereto, we make use of a global anatomical affine optical flow method (Barbosa et al., 2013b), which allows performing in-plane tracking of the myocardial motion during the cardiac cycle.

Using the resulting delineations, one is able to compute several clinical cardiac indices, such as end-diastolic volume (EDV), endsystolic volume (ESV), stroke volume (SV), LVM and EF, in an automated way.

\subsection{Automatic initialization using an elliptical annular template matching algorithm}

In Queirós et al. (2013), we introduced a new automatic initialization algorithm for a mid-ventricular slice based on a LV localization method and an elliptical annular template matching algorithm.

In brief, the method detects the LV centroid by applying a multilevel Otsu thresholding (Otsu, 1975) to divide the image in four classes (blood, soft tissues, background and one related to bright artifacts), followed by a class decomposition and a search procedure among the candidate objects. To this end, the 4 classes are used to create 3 independent images to be analyzed (Fig. 2B), taking into account their possible distribution in the presence or absence of artifacts: an image with the brighter class alone (since the blood pool is usually the brightest object in the image); an image with the two brightest classes combined (in the presence of artifacts or due to heterogeneities related to blood flow, the cavity can be divided between these classes); and an image with only the third brightest class (in case strong artifacts occupy the two brightest classes). The LV object is chosen as the most circular and close to the image's center among the three analyzed images (Fig. 2C) (Queirós et al., 2013).

In a second step, in order to obtain two elliptical initial contours to be used in the segmentation procedure, a template matching method designed to search for a dark elliptical ring is applied (Queirós et al., 2013). The strategy is to find the template (by varying the ring's major axis, thickness, orientation and eccentricity in a total of 240 possibilities - Fig. 2D) that best fits the subject-specific myocardium (Fig. 2E). Note that to reduce the search space, the object detected in the first step is used to fit an ellipse and estimate the major axis and orientation, which defines the central value of the search range for these parameters. For every kernel, a block matching search is performed using normalized cross correlation and the position maximizing the cross correlation value defines the optimal center for the kernel under evaluation. Finally, the kernel having the maximum cross correlation value among all kernels analyzed defines the optimal parameters, used to draw two ellipses to be used as initial contours (Fig. 2F and G) (Queirós et al., 2013). 

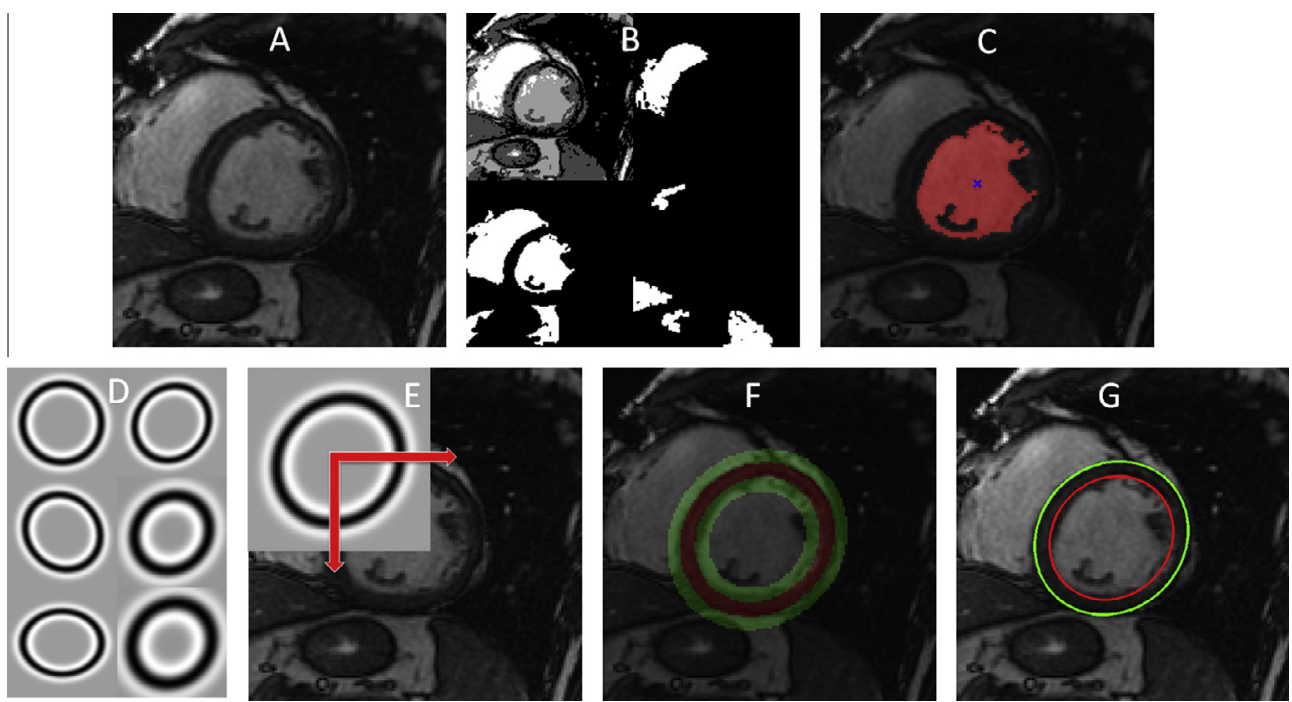

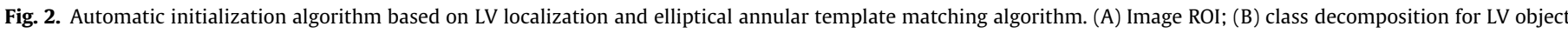

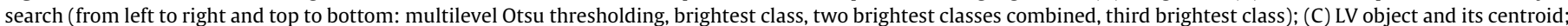

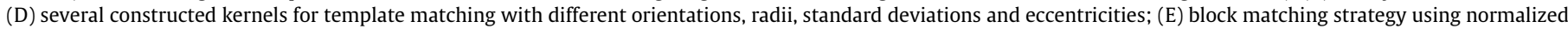
cross correlation, (F) original image overlaid with optimal kernel; and (G) resulting elliptical initial contours.

\subsection{Coupled myocardial $2 D$ segmentation}

\subsubsection{B-Spline Explicit Active Surfaces}

The key concept of the BEAS framework is to regard the boundary of an object as an explicit function, where one of the coordinates of the points within the surface, $\mathbf{x}=\left\{x_{1}, \ldots, x_{n}\right\}$, is given explicitly as a function of the remaining coordinates, i.e. $x_{1}=\psi\left(x_{2}, \ldots, x_{n}\right)$. In this framework (Barbosa et al., 2012), $\psi$ is defined as a linear combination of B-spline basis functions:

$x_{1}=\psi\left(x_{2}, \ldots, x_{n}\right)=\psi\left(\mathbf{x}^{*}\right)=\sum_{\mathbf{k} \in \mathbb{Z}^{n-1}} c[\mathbf{k}] \beta^{d}\left(\frac{\mathbf{x}^{*}}{h}-\mathbf{k}\right)$,

where $\beta^{d}(\cdot)$ is the uniform symmetric $(n-1)$-dimensional B-spline of degree $d$. The knots of the B-splines are located on a rectangular grid defined on the chosen coordinate system, with a regular spacing given by $h$. The coefficients of the B-spline representation are gathered in $c[\mathbf{k}]$.

\subsubsection{Endocardial and epicardial contour coupling}

In typical active contour formulations, the endo and epicardial boundaries are defined separately and additional penalties guarantee their coupling (Paragios, 2002; Chen et al., 2008). Inspired by the strategy used in the early work of Barbosa et al. (2010) for the LV segmentation in US, we model the endo and epicardial contours as a combination of two explicit functions representing the myocardial wall position $\left(\psi_{W_{p}}(\theta)\right)$ and wall thickness $\left(\psi_{W_{T}}(\theta)\right)$, respectively. Thereby, each contour is defined as:

$\Gamma_{\text {endo }}(\theta)=\psi_{W_{P}}(\theta)-\psi_{W_{T}}(\theta)$,

$\Gamma_{\text {epi }}(\theta)=\psi_{W_{P}}(\theta)+\psi_{W_{T}}(\theta)$,

where $\psi_{W_{p}}(\theta)$ gives the position of the myocardial wall as a function of $\theta$ and $\psi_{W_{T}}(\theta)$ encodes half of the myocardial wall thickness. Both $\Gamma_{\text {endo }}$ and $\Gamma_{\text {epi }}$ act as interfaces between the blood pool (in), the myocardium (myo) and the surrounding structures (out). The mathematical derivation that supports the variational formulation of this coupling strategy, not included in the early work of Barbosa et al. (2010), will be presented in Section 2.3.4 and Appendix A. Moreover, the corresponding extension to a 3D formulation will be proposed in Section 2.5.1.
The fundamental advantage of such formulation is that, by applying different scales ( $h$ in Eq. (1)) to $\psi_{W_{P}}$ and $\psi_{W_{T}}$, one can implicitly smooth the local variation of the myocardial thickness, in order to keep it smooth, without affecting the overall ability to capture more complex myocardial shapes. Moreover, it allows global shape constraints to be applied as shape penalties in the energy functional and controlling the coupled segmentation instead of each separated contour (Barbosa et al., 2010).

\subsubsection{Proposed $2 D$ energy functional}

As commonly used in active contours methodologies (Lankton and Tannenbaum, 2008), our proposed energy functional is expressed as the sum of a data attachment term, $E_{d}$, and a regularization term, $E_{r}$, therefore being expressed as:

$$
E=E_{d}+E_{r}
$$

Since our model is defined based on the myocardial wall position and thickness and given its B-spline formulation, it can be shown that the energy functional is directly minimized through the optimization of the B-spline coefficients $c[\mathbf{k}]$ (Barbosa et al., 2012). Therefore, the energy minimization process can be achieved using the following evolution equations:

$\frac{\partial E}{\partial c_{W_{P}}\left[\mathbf{k}_{\mathbf{j}}\right]}=\frac{\partial E_{d}}{\partial c_{W_{P}}\left[\mathbf{k}_{\mathbf{j}}\right]}+\frac{\partial E_{r}}{\partial c_{W_{P}}\left[\mathbf{k}_{\mathbf{j}}\right]}$

$\frac{\partial E}{\partial c_{W_{T}}\left[\mathbf{k}_{\mathbf{j}}\right]}=\frac{\partial E_{d}}{\partial c_{W_{T}}\left[\mathbf{k}_{\mathbf{j}}\right]}+\frac{\partial E_{r}}{\partial c_{W_{T}}\left[\mathbf{k}_{\mathbf{j}}\right]}$

\subsection{4. $2 \mathrm{D}$ data attachment term}

Lankton and Tannenbaum (2008) have recently introduced the concept of localized region-based energies, which overcome the limitations of classical edge-based and global region-based energy functionals. In the present work, we propose to use two different localized region-based energies for the endo and epicardial contours, which take the specific local appearance of each boundary into account. Since the blood pool is brighter than the myocardial tissue, a signed version of the localized Yezzi (LY) energy (Lankton and Tannenbaum, 2008; Barbosa et al., 2013a) is used for the endocardial contour (henceforward referred as signed localized Yezzi - 


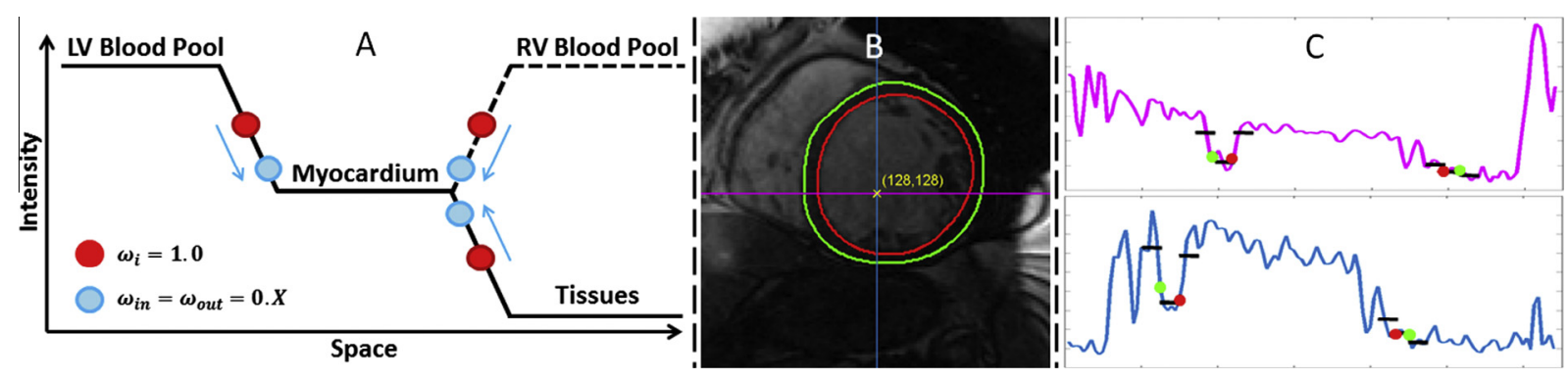

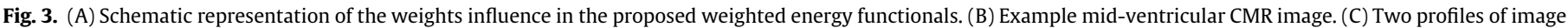

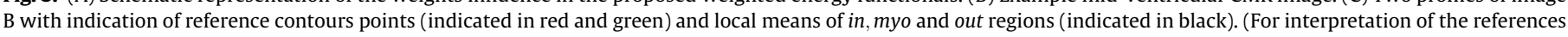
to colour in this figure legend, the reader is referred to the web version of this article.)

SLY). This energy explicitly introduces the expected intensity relation between the in and myo regions, forcing the contour to evolve towards an interface whose internal region is brighter than the external one. On the other hand, since the external region (out) is relatively unpredictable due to the encountered variability in structures (e.g., lungs, RV or soft tissues - Fig. 2A), an unsigned constant intensity model is chosen by using the localized ChanVese (LCV) energy (Lankton and Tannenbaum, 2008).

Additionally, these localized energy functionals were modified by adding weights to the inner and outer regions of the endocardial and epicardial interfaces, $w_{\text {in }}$ and $w_{\text {out }}$, respectively. This formulation allows an explicit control over the equilibrium point in between the two regions, therefore pushing the contour towards the myocardial region if a weight smaller than one is considered (see Fig. 3A). In practice, this strategy mimics the physician's knowledge when manually contouring, where they place the contours closer to the myocardium to avoid TPMs to be included in this region, as illustrated in Fig. 3B and C.

Given the topology of the segmentation problem, the proposed energy functional is defined as:

$$
\begin{aligned}
E_{d}= & \int_{\Omega} \delta_{\phi_{\text {endo }}}(\mathbf{x}) \int_{\Omega} B(\mathbf{x}, \mathbf{y}) \cdot F_{\text {endo }}(\mathbf{y}) d \mathbf{y} d \mathbf{x} \\
& +\int_{\Omega} \delta_{\phi_{\text {epi }}}(\mathbf{x}) \int_{\Omega} B(\mathbf{x}, \mathbf{y}) \cdot F_{\text {epi }}(\mathbf{y}) d \mathbf{y} d \mathbf{x},
\end{aligned}
$$

where

$F_{\text {endo }}(\mathbf{y})=u_{x, m y o}-w_{\text {in }} \cdot u_{x, \text { in }}$,

$F_{\text {epi }}(\mathbf{y})=f_{\text {myo }}(y) \cdot\left(H_{\phi_{\text {epi }}}(\mathbf{y})-H_{\phi_{\text {endo }}}(\mathbf{y})\right)+f_{\text {out }}(y) \cdot\left(1-H_{\phi_{\text {epi }}}(\mathbf{y})\right)$,

$f_{\text {myo }}(\mathbf{y})=\left(I(\mathbf{y})-u_{x, \text { myo }}\right)^{2}$,

$f_{\text {out }}(\mathbf{y})=w_{\text {out }} \cdot\left(I(\mathbf{y})-u_{x, \text { out }}\right)^{2}$,

and $w_{i}$ is the scalar weight applied to the region $i ; u_{x, i n}, u_{x, m y o}$ and $u_{x, \text { out }}$ represent the local intensity means in the vicinity of $x$ for the inner, myocardial and outer regions, respectively, as defined in Lankton and Tannenbaum (2008); and $I(\mathbf{y})$ is the image intensity at position $\mathbf{y} . H_{\phi_{j}}$ is the Heaviside operator applied to the level-set like function $\phi_{j}(\mathbf{x})=\Gamma_{j}\left(\mathbf{x}^{*}\right)-x_{1}$, representing the region inside the interface $j . B(\mathbf{x}, \mathbf{y})$ corresponds to a mask function in which the local parameters that drive the evolution of the interfaces are estimated. In the present work, in order to maintain low computational costs, we chose to use a squared region centered in $\mathbf{x}$, whose size will be discussed in Section 4.

Given the derivation available in Appendix A, the minimization of the data attachment term can be achieved using the following evolution equations:

$$
\frac{\partial E_{d}}{\partial c_{W_{P}}\left[\mathbf{k}_{\mathbf{j}}\right]}=\int_{\Gamma_{\text {endo }}} \bar{g}_{\text {endo }}\left(\mathbf{x}^{*}\right) \beta^{d}\left(\frac{\mathbf{x}^{*}}{h}-\mathbf{k}_{\mathbf{j}}\right) d \mathbf{x}^{*}+\int_{\Gamma_{\text {epi }}} \bar{g}_{\text {epi }}\left(\mathbf{x}^{*}\right) \beta^{d}\left(\frac{\mathbf{x}^{*}}{h}-\mathbf{k}_{\mathbf{j}}\right) d \mathbf{x}^{*}
$$

$$
\begin{aligned}
\frac{\partial E_{d}}{\partial c_{W_{T}}\left[\mathbf{k}_{\mathbf{j}}\right]}= & -\int_{\Gamma_{\text {endo }}} \bar{g}_{\text {endo }}\left(\mathbf{x}^{*}\right) \beta^{d}\left(\frac{\mathbf{x}^{*}}{h}-\mathbf{k}_{\mathbf{j}}\right) d \mathbf{x}^{*} \\
& +\int_{\Gamma_{\text {epi }}} \bar{g}_{\text {epi }}\left(\mathbf{x}^{*}\right) \beta^{d}\left(\frac{\mathbf{x}^{*}}{h}-\mathbf{k}_{\mathbf{j}}\right) d \mathbf{x}^{*}
\end{aligned}
$$

where

$\bar{g}_{\text {endo }}\left(\mathbf{x}^{*}\right)=\left(\frac{\bar{I}\left(\mathbf{x}^{*}\right)-w_{\text {myo }} \cdot u_{x, m y o}}{A_{u_{\text {myo }}}}\right)-\left(\frac{\bar{I}\left(\mathbf{x}^{*}\right)-w_{\text {in }} \cdot u_{x, i n}}{A_{u_{\text {in }}}}\right)$,

$\bar{g}_{\text {epi }}\left(\mathbf{x}^{*}\right)=\bar{f}_{\text {myo }}\left(\mathbf{x}^{*}\right)-\bar{f}_{\text {out }}\left(\mathbf{x}^{*}\right)$.

and $A_{u_{\text {myo }}}$ and $A_{u_{i n}}$ are the areas of the myocardial and inner regions used to estimate the local means $u_{x, m y o}$ and $u_{x, i n}$, respectively (Lankton and Tannenbaum, 2008). Moreover, considering a generic function $h(x)$ in $\mathbb{R}^{n}$, we note $\bar{h}$ as the restriction of $h$ over the interface $\Gamma$ in $\mathbb{R}^{(n-1)}$ (c.f. Eq. (A.10)). Thus, $\bar{I}\left(\mathbf{x}^{*}\right)$ corresponds to the image value at position $\mathbf{x}=\left\{x_{1}=\psi\left(\mathbf{x}^{*}\right), x_{2}, \ldots, x_{n}\right\}$.

\subsection{5. $2 D$ Regularization term}

Since it is possible that both contours are attracted towards the same image feature leading to their merging, a thickness penalty is included in the evolution equations of the coupled segmentation problem. Such regularization term prevents the contours' merging by locally penalizing a myocardial thickness of less than $1 p x$. Let us note that in Chen et al. (2008), the authors also introduced a thickness constraint in the energy, but they considered it as a global term (a deviation from the average thickness) modeled as two distinct level-sets and not considering its local variation. Since the term introduced in Chen et al. (2008) favors constant thickness, which limits the flexibility of the model to deal with pathological cases, the formulation introduced in Dietenbeck et al. (2011) was followed instead. However, the term introduced in Dietenbeck et al. (2011) has an "on-off" behavior below the minimum thickness, whereas a more gradual penalization of small thicknesses would be desirable. To this end, we have modified the term introduced in Dietenbeck et al. (2011), thus expressing the thickness regularization term as:

$E_{T}=\frac{1}{2} \int_{\Omega} \phi\left(\mathbf{x}+R_{T} \mathbf{N}\right)^{2} H\left(\phi\left(\mathbf{x}+R_{T} \mathbf{N}\right)\right) \delta(\phi(\mathbf{x})) d \mathbf{x}$,

where $\mathbf{N}$ corresponds to the inward normal of a point $\mathbf{x}$ belonging to the contour implicitly defined by the level-set function $\phi(\mathbf{x})$ and $R_{T}$ is the thickness value below which the regularization term is activated. Following the derivation presented in Dietenbeck et al. (2011), and given that $\frac{\partial}{\partial \phi} \phi(\mathbf{y})^{2}=2 \phi(\mathbf{y})$, the variation of this energy term wrt. $\phi$ can be expressed as:

$\frac{\partial E_{T}}{\partial \phi}=\phi\left(\mathbf{x}+R_{T} \mathbf{N}\right) H\left(\phi\left(\mathbf{x}+R_{T} \mathbf{N}\right)\right) \delta(\phi(\mathbf{x}))$. 

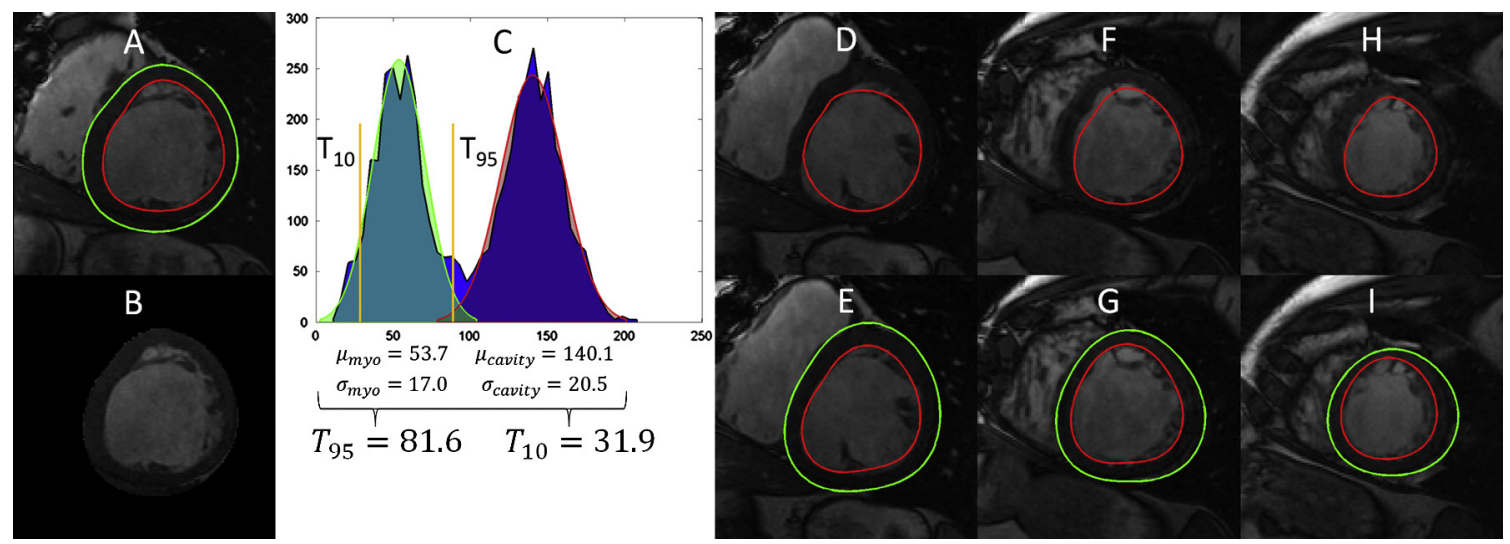

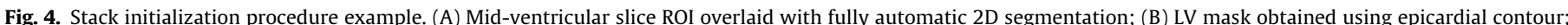

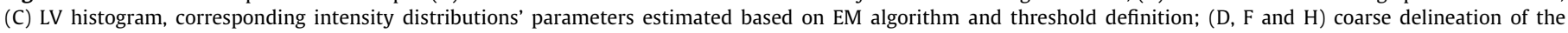
endocardium using threshold-based BEAS; and (E, G and I) final initialization for both endo and epicardial contours using coupled threshold-based BEAS.

Thanks to the coupled formulation here presented, the term $\phi\left(\mathbf{X}+R_{T} \mathbf{N}\right)$ can be simplified since the local (half) thickness of the myocardium is explicitly defined by $\psi_{W_{T}}\left(\mathbf{x}^{*}\right)$. Thus, the term $\phi\left(\mathbf{x}+R_{T} \mathbf{N}\right)$ can be simply expressed as $\left(R_{T}-2 * \psi_{W_{T}}\left(\mathbf{x}^{*}\right)\right)$. Note that as $\phi\left(\mathbf{x}+R_{T} \mathbf{N}\right)>0$ only if the local thickness is smaller than $R_{T}$, also the term $\left(R_{T}-2 * \psi_{W_{T}}\left(\mathbf{x}^{*}\right)\right)$ is equally positive only when half of the thickness, $\psi_{W_{T}}\left(\mathbf{x}^{*}\right)$, is smaller than $R_{T} / 2$. Taking into account the details presented in the Appendix, namely (A.7), (A.8) and (A.10), the thickness regularization term can be used to directly update the B-spline coefficients controlling the myocardial thickness using:

$\frac{\partial E_{T}}{\partial c_{W_{T}}\left[\mathbf{k}_{\mathbf{i}}\right]}=\int\left(R_{T}-2 * \psi_{W_{T}}\left(\mathbf{x}^{*}\right)\right) H\left(R_{T}-2 * \psi_{W_{T}}\left(\mathbf{x}^{*}\right)\right) \beta^{d}\left(\frac{\mathbf{x}^{*}}{h}-\mathbf{k}_{\mathbf{i}}\right) d \mathbf{x}^{*}$.

\subsection{Automatic stack initialization}

The previous sections focused on obtaining an accurate and automatic segmentation for a mid-ventricular slice. However, the goal is to segment all SAX slices from base to apex, which demands an initialization on these slices. In this section, an automatic 3D stack initialization is proposed.

The simplest initialization would consist of naively using the segmentation result at the mid-ventricular level as initial contours of other slices in the stack, plus a possible scaling to account for different sizes of the LV SAX cuts from base to apex. However, this approach makes two unreliable assumptions: it first considers that the relative LV size in different slices is known or can be estimated a priori; moreover, it assumes the cavity center is always placed in the same position, which may not be true due to the known misalignment of the slices in CMR datasets.

Thus, we propose a threshold-based BEAS formulation to initialize both endo and epicardial contours in the full stack, while at the same time estimating possible slice misalignment.

The idea is to first estimate the expected intensity distribution of the LV cavity and myocardial wall based on the previous 2D segmentation. We propose to use an Expectation-Maximization (EM) algorithm (Moon, 1996) assuming a mixture of two gaussians to describe the LV (cavity plus myocardium) histogram. After estimating the intensity distribution parameters (mean and standard deviation), two thresholds are defined, namely the minimum cavity intensity and the minimum myocardium intensity (Fig. 4C). Due to the presence of TPMs and because they should be included in the blood pool, the cavity's distribution is discarded and only the myocardial one is used to define both thresholds. The cavity intensity threshold is defined as the 95 percentile of the myocardial dis- tribution, which is a sufficiently high value to capture the full blood pool plus TPMs, while simultaneously avoiding the contour's leakage to the myocardium. On the other hand, the myocardium intensity threshold is defined as the 10 percentile of the myocardial distribution, allowing to capture the myocardial wall and avoid the leakage to darker regions (such as the lungs). In Section 5, the choice of these thresholds is addressed and the influence of their variation is investigated.

To obtain a coarse delineation of the endocardium, we start by placing an initial circular contour with a small radius ( $2 p x)$ centered in the LV cavity's center of the mid-ventricular slice and evolve it using the minimum cavity threshold. In this sense, if the intensity of the contour point is higher than the threshold, the contour is allowed to grow. In contrast, when it is lower than the threshold, the contour is shrunk (Shi and Karl, 2008). Moreover, at each iteration, an additional step is done focusing on re-initializing the center's position to be the contours' center of gravity and consequently resample the contour in the polar domain. The idea is to better estimate the cavity's center for each slice independently. Finally, the evolution stops when the area enclosed by the contour does not significantly change for a few iterations.

We then initialize an epicardial contour with the thickness obtained in the 2D mid-ventricular segmentation and evolve it using the minimum myocardium threshold. To improve the initialization robustness, the coupling strategy described in Section 2.3.2 is used in this second stage. This allows the epicardial contour delineation, while also enabling a better endocardial definition by surpassing possible errors in the first stage. Again, the evolution stops when the area enclosed by both contours stays nearly constant for a few iterations.

The proposed method is applied to each slice in the stack (Fig. 4D-I), progressively using the LV cavity's center from previously initialized adjacent slices to start the endocardial contour. In the end, it provides a consistent initialization for the subsequent segmentation method, while estimating the slice misalignment.

\subsection{Coupled myocardial 3D segmentation}

\subsubsection{BEAS 3D extension}

The proposed automatic initialization procedure allows to obtain an initial contour plus an estimate for the LV cavity's center for each slice. Using such initialization, a segmentation could be independently applied in each slice. However, it would not take into account the known spatial continuity of the LV. Thus, a 3D 

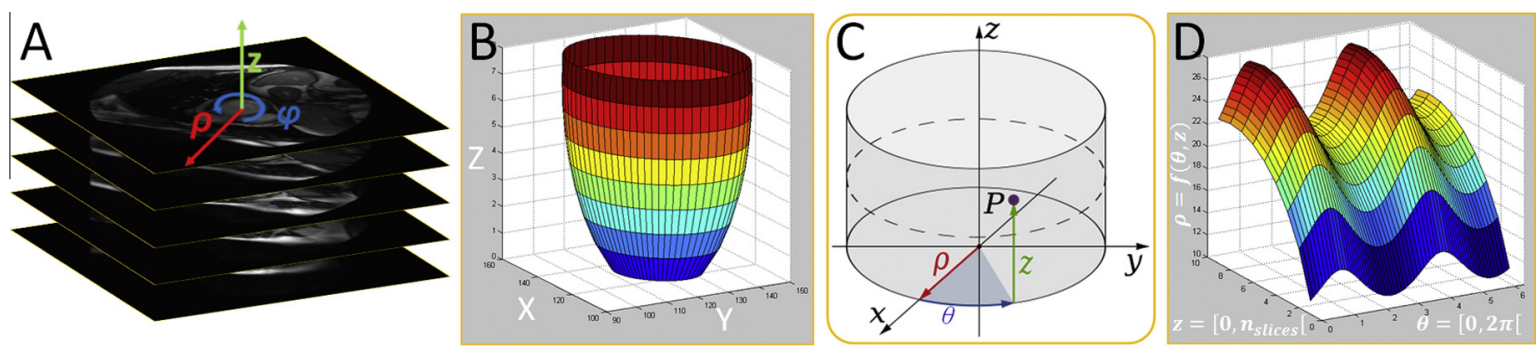

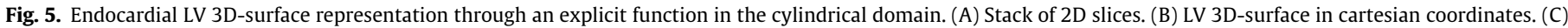
Correspondence between the cartesian and cylindrical domains. (D) Explicit function in the cylindrical domain.
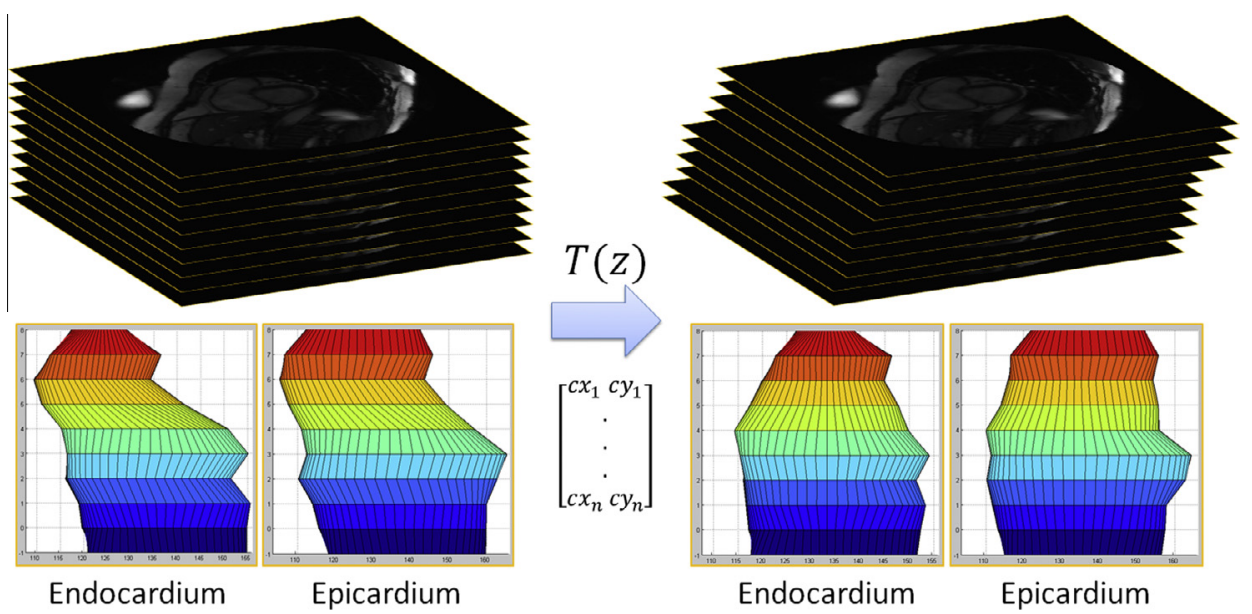

Fig. 6. Misalignment correction based on translation applied according to the estimated LV cavity's centers of each slice.

segmentation enforcing a spatial continuity allows a more robust segmentation, minimizing possible errors in individual slices.

In the BEAS framework, an important step towards this 3D modeling is the choice of an appropriate coordinate system. As the CMR slices have a relatively large thickness and the pixel intensities between slices cannot be reliably estimated, considering a spherical coordinate system (which would be the most straightforward BEAS extension) is unfeasible. Therefore, we propose to use a cylindrical coordinate system, where the third dimension has only a finite number of possible values corresponding to the number of slices in the stack (Fig. 5). At the same time, we improved the formulation introduced in Barbosa et al. (2010) (Section 2.3.2) for an implicit coupled propagation by extending it to a three-dimensional parameterization of the myocardium, making use of the cylindrical topology of the CMR data. Thus, the 3D extension of Eqs. (2) and (3) yields:

$\Gamma_{\text {endo }}(\theta, z)=\psi_{W_{P}}(\theta, z)-\psi_{W_{T}}(\theta, z)$,

$\Gamma_{e p i}(\theta, z)=\psi_{W_{P}}(\theta, z)+\psi_{W_{T}}(\theta, z)$

where $\psi_{W_{P}}(\theta, z)$ gives the position of the myocardial wall as a function of $\theta$ and the slice level $z$, and $\psi_{W_{T}}(\theta, z)$ encodes half of the myocardial wall thickness. One should note that the proposed formulation only allows the surface points to evolve radially, inside its own initial $z$ level. Such constraint is crucial as any inter-slice information is unknown.

Moreover, several points are important to ensure a proper surface evolution. On the one hand, extending the neighborhood to a cubic local volume would lead to incorrect local statistics calculations due to the data anisotropy and considerable slice thickness. As such, we continue to use an in-slice squared region to update the local means around each interface. On the other hand, the introduction of a new dimension requires an additional B-spline scale to be defined in the formulation. However, it has to be a low scale to avoid over-smoothing of the 3D surface in the third dimension, as a reduced number of slices are acquired to cover the left ventricle.

Importantly, one should note the spatial continuity of the cylindrical parametric representation might be affected in case of slice misalignment due to the smooth nature of our B-spline representation. Therefore, in order to represent the LV surface with a consistent cylindrical topology and to allow for spatial continuity, we propose to reduce the slice misalignment prior to the 3D segmentation by considering the LV cavity's centers estimated during the automatic stack initialization (Section 2.4). To this end, a translation is applied to each slice correcting the center's position, resulting in a virtually straight LV axis, as illustrated in Fig. 6. Such alignment allows for a synergistic interaction between the segmented contours in neighboring slices during surface evolution, therefore enabling a truly three-dimensional analysis of the data without compromising the performance of the proposed 3D segmentation algorithm.

\subsubsection{D Data attachment term}

Due to the contrast in MR images and subsequent well-defined edges, several authors use edge-based energy terms to evolve the contours and segment the LV (Paragios, 2002; Pluempitiwiriyawej et al., 2005; Chen et al., 2008). Although such edges are well defined at the endocardium, they usually lead to wrong contouring when TPMs are present unless another strategy is added. Regarding the epicardial delineation, even with low contrast between myocardium and surrounding structures (e.g., lungs), edge-based terms can substantially help if properly included. Taking this into account, we propose to use a hybrid formulation for the epicardial energy functional 

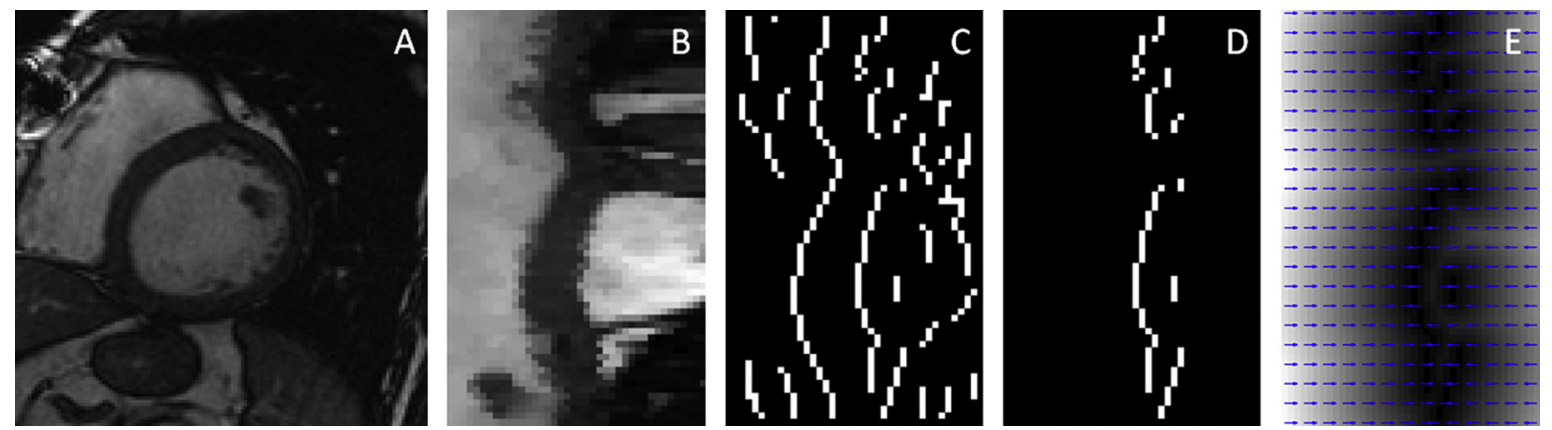

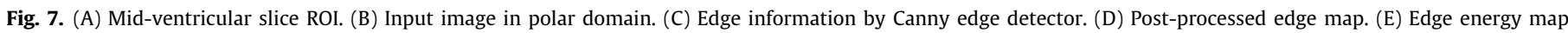
overlaid with radial vector field.

by introducing an edge-based term. This term is only applied to the epicardium, because the presence of TPMs in the blood pool may mislead the contours' evolution due to additional edges. Furthermore, as a result of higher contrast between cavity and muscle, the segmentation result in the endocardium is expected to be better than the epicardial one. The proposed edge-based term relies on the computation of an edge map and its subsequent post-processing. Inspired by the method in Lee et al. (2010), for each slice in the ED phase an edge map is computed following 4 steps:

1. The image is mapped to polar space using the LV cavity's center estimated during the automatic stack initialization (Fig. 7B).

2. A Canny edge detector (Canny, 1986) is applied to extract the edge information (Fig. 7C). Note that the high threshold was defined in order to obtain $60 \%$ of the edge information, while the lower threshold was 0.4 times the high one.

3. A post-processing step is applied to filter the edge information (Fig. 7D). Specifically, the initial endocardial contour is used to filter out any edge belonging to the cavity's boundary or inner region. Moreover, the edges in more remote regions are also cleaned as they are associated with a myocardium thickness that is unlikely (over $20 \mathrm{~mm}$ ).

4. The edge energy map is computed by calculating the unsigned distance of every image's pixel to the closest edge point (Fig. 7E). This distance function allows to improve the edge detection robustness by filling small gaps in missed edges.

Using the resulting edge energy map, $\Upsilon(\mathbf{y})$, as a new term in the epicardial energy, the complete proposed energy functional for 3D segmentation is defined as:

$$
\begin{aligned}
E_{d}= & \int_{\Omega} \delta_{\phi_{\text {endo }}}(\mathbf{x}) \int_{\Omega} B(\mathbf{x}, \mathbf{y}) \cdot F_{\text {endo }}(\mathbf{y}) d \mathbf{y} d \mathbf{x} \\
& +\int_{\Omega} \delta_{\phi_{\text {epi }}}(\mathbf{x}) \int_{\Omega} B(\mathbf{x}, \mathbf{y}) \cdot\left(F_{\text {epi }}(\mathbf{y})+\alpha \cdot \Upsilon(\mathbf{y})\right) d \mathbf{y} d \mathbf{x},
\end{aligned}
$$

where $\alpha$ is a positive hyper-parameter to balance the two terms in the epicardial energy.

The energy minimization process can be achieved using the following evolution equations:

$$
\begin{aligned}
\frac{\partial E_{d}}{\partial c_{W_{P}}\left[\mathbf{k}_{\mathbf{i}}\right]}= & \int_{\Gamma_{\text {endo }}} \bar{g}_{\text {endo }}\left(\mathbf{x}^{*}\right) \beta^{d}\left(\frac{\mathbf{x}^{*}}{h}-\mathbf{k}_{\mathbf{i}}\right) d \mathbf{x}^{*} \\
& +\int_{\Gamma_{\text {epi }}}\left(\bar{g}_{\text {epi }}\left(\mathbf{x}^{*}\right)+\alpha \cdot \nabla_{r} \bar{\Upsilon}\left(\mathbf{x}^{*}\right)\right) \beta^{d}\left(\frac{\mathbf{x}^{*}}{h}-\mathbf{k}_{\mathbf{i}}\right) d \mathbf{x}^{*},
\end{aligned}
$$

$$
\begin{aligned}
\frac{\partial E_{d}}{\partial c_{W_{T}}\left[\mathbf{k}_{\mathbf{i}}\right]}= & -\int_{\Gamma_{\text {endo }}} \bar{g}_{\text {endo }}\left(\mathbf{x}^{*}\right) \beta^{d}\left(\frac{\mathbf{x}^{*}}{h}-\mathbf{k}_{\mathbf{i}}\right) d \mathbf{x}^{*} \\
& +\int_{\Gamma_{\text {epi }}}\left(\bar{g}_{\text {epi }}\left(\mathbf{x}^{*}\right)+\alpha \cdot \nabla_{r} \bar{\Upsilon}\left(\mathbf{x}^{*}\right)\right) \beta^{d}\left(\frac{\mathbf{x}^{*}}{h}-\mathbf{k}_{\mathbf{i}}\right) d \mathbf{x}^{*},
\end{aligned}
$$

where $\nabla_{r} \bar{\Upsilon}$ is the edge energy map gradient along the radial direction.

\subsubsection{D Regularization term}

Similarly to the 2D case, the 3D regularization term comprehends a thickness term for contours' merging prevention.

Moreover, even though the B-spline support of the coupled contours ensures a smooth interface, strong image features near the papillary muscles can still wrongly attract the endocardial contour. To avoid this, a curvature-based shape prior is also included as a regularization term in the endocardial energy functional to avoid concave shapes. This can be easily achieved using a binary curvature-based switch that locally controls if the endocardial contour should evolve according to the image features or if it should rather be regularized to avoid concave shapes. The proposed shape regularization strategy was simply implemented making use of a conventional mean curvature flow term, as the one used in Chan and Vese (2001), which is only applied to regions of negative curvature. Thus, the endocardial feature function was modified by:

$\hat{g}_{\text {endo }}\left(\mathbf{X}^{*}\right)=H\left(\kappa_{\text {endo }}\left(\mathbf{x}^{*}\right)\right) \bar{g}_{\text {endo }}\left(\mathbf{x}^{*}\right)-H\left(-\kappa_{\text {endo }}\left(\mathbf{x}^{*}\right)\right) \kappa_{\text {endo }}\left(\mathbf{x}^{*}\right)$,

where $\kappa_{\text {endo }}\left(\mathbf{x}^{*}\right)$ is the local curvature on the endocardial contour. Note that the binary weighting term $H\left(-\kappa_{\text {endo }}\left(\mathbf{x}^{*}\right)\right)$ constrains the curvature regularization to concave regions in the segmented endocardial interface, whereas $H\left(\kappa_{\text {endo }}\left(\mathbf{x}^{*}\right)\right)$ allows to normally evolve the endocardial contour according to the segmentation functional introduced in (21). The relevance of this regularization term in the proposed 3D segmentation energy is discussed in Section 6.

\subsection{D+time Tracking using anatomical affine optical flow}

In the previous sections, a 3D segmentation for all slices covering the LV was obtained for the ED phase. In order to compute the left ventricular EF, one requires the segmentation to be performed in both ED and ES phases. Several approaches can be used for this purpose, namely temporal segmentation or tracking procedures. The pure segmentation-based approaches focus on identifying the relevant object on consecutive images, using specific energy terms for temporal integration (Lynch et al., 2008). In contrast, tracking-based approaches normally focus on following the motion of specific patterns along the sequence of images (Hautvast et al. 2006).

We propose to use a tracking algorithm to segment the LV in the whole cardiac cycle, by estimating the motion between adjacent cardiac phases (starting from the previously segmented contours in the ED phase). Because CMR data is highly anisotropic and the motion cannot be reliably estimated in the third dimension, each slice sequence should be independently analyzed. Moreover, as both endo and epicardial contours need to be tracked through all 
phases, the motion estimation should be performed independently for each boundary as well.

In this sense, the motion estimation is performed using the global anatomical affine optical flow method proposed by Barbosa et al. (2013b), firstly presented for three-dimensional US data. The principle is to estimate an anatomically constrained global affine transform between two consecutive frames by taking into account the estimated motion over the reference contour points in the previous frame. Such constrained estimation only considers anatomical regions of interest and avoids the influence of adjacent tissues. Moreover, by including an iterative displacement refinement scheme, the algorithm is still able to accurately capture large displacements (Barbosa et al., 2013b).

Let $I\left(x_{1}, x_{2}, t\right)$ denote the pixel intensity at location $\mathbf{x}=\left[x_{1}, x_{2}\right]$ and time $t$ for a given slice temporal sequence. Based on the least square solution of the optical flow equation, the 2D affine motion (for each slice and each boundary) can be estimated by minimizing the following energy:

$E_{M}=\int_{\mathbb{R}^{2}} \mathcal{W}\left(x_{1}-c_{1}, x_{2}-c_{2}\right)\left(I_{x_{1}} u+I_{x_{2}} v+I_{t}\right)^{2} d \mathbf{x}$,

where

$u\left(x_{1}, x_{2}\right)=u_{0}+u_{x_{1}}\left(x_{1}-c_{1}\right)+u_{x_{2}}\left(x_{2}-c_{2}\right)$,

$v\left(x_{1}, x_{2}\right)=v_{0}+v_{x_{1}}\left(x_{1}-c_{1}\right)+v_{x_{2}}\left(x_{2}-c_{2}\right)$,

encode the local motion field along $x_{1}$ and $x_{2}$ respectively. From such estimated motion field, the affine transformation to be applied to the analyzed slice and contour can be written as the augmented matrix:

$A=\left[\begin{array}{ccc}1+u_{x_{1}} & u_{x_{2}} & u_{0} \\ v_{x_{1}} & 1+v_{x_{2}} & v_{0} \\ 0 & 0 & 1\end{array}\right]$,

$\nabla I=\left[I_{x_{1}}, I_{x_{2}}\right]$ is the local image spatial gradient, whereas $I_{t}$ corresponds to the temporal derivative. In order to reduce the effect of noise in the assessment of the spatial gradient, $\nabla I(\mathbf{x})$ was computed using gaussian derivative kernels along $x_{1}$ and $x_{2}$, with $\sigma=1$. $I_{t}$ was simply estimated with finite forward differences. $\mathcal{W}$ is a local window function centered in the position $\mathbf{c}=\left[c_{1}, c_{2}\right]$ and, according to the anatomical constraint introduced in Barbosa et al. (2013b), is given by:

$\mathcal{W}(\mathbf{x})=\sum_{x_{k} \in \Gamma} \mathcal{N}\left(\mathbf{x}_{\mathbf{k}}\right)$,

where $\mathcal{N}(\mathbf{x})$ is a neighborhood function defined as a 2D square center in $\mathbf{x}$ and $x_{k} \in \Gamma$ stands for the sampled points in the LV contour being tracked. $\mathcal{N}$ was defined as a $7 \times 7$ square centered in the target point. Such region is smaller than the one in US data (Barbosa et al., 2013b) due to the larger pixel spacing in CMR images and reduced uncertainty associated with this data. To decrease the method's computational burden, we propose to subsample the contours by a factor of 4 , reducing the required local motion estimations. Such subsampling is possible because superposition of local regions can be avoided without losing robustness for the global estimation.

The proposed pure tracking-based energy is used to find the global affine transformation between consecutive frames, independently applied to each SAX cut and each boundary. Using such principle, the propagation is applied sequentially between adjacent phases using the previous LV contours, until every cardiac phase has been tracked. Note that to minimize error accumulation, we ensure a minimal number of propagation steps by propagating the contours in both directions for half the total number of cardiac phases.

\section{Implementation details}

Concerning BEAS-related parameters in the 2D segmentation, the B-spline scale, $h$, was set to $2^{2}$ and $2^{3}$ for the wall position and thickness respectively, while for the $3 \mathrm{D}$ segmentation a value of $2^{3}$ and $2^{4}$ was used. The increased spacing allows a higher degree of smoothness of the surface's shape and thickness, which helps overcoming possible errors due to the presence of TPMs or in cases of low contrast and reduced spatial information. Regarding the smoothing across slices, a value of $2^{0}$ was set. Similar to Queirós et al. (2013), 64 points per slice were used on each boundary for the contour discrete representation. Furthermore, a local region of size $15 \times 15$ and $11 \times 11$ was used to assess the local region means for the endo and epicardial contours, respectively. The local region is smaller for the epicardium because of the unpredictability of the outer region, which may introduce errors in the local mean estimation. The proposed region weights, $w_{i}$, were set to 0.3 for both in and out regions. As stated in Section 2.3.3, a value lower than one pushes the contours towards the myocardial region, surpassing the presence of TPMs. The chosen values allow a good compromise for contour delineation, without leakage into the myocardial region. At last, the parameter $\alpha$ in the hybrid epicardial energy functional for 3D segmentation was empirically set to 0.05 . For further general considerations and implementation details regarding BEAS, the reader is referred to the work of Barbosa et al. (2012). In Section 5, the choice of these parameters is addressed and the influence of their variation in the results is investigated.

\section{Validation experiments}

To assess the performance of the proposed framework, we used the cine steady state free precession (SSFP) MR SAX datasets available from the MICCAI 2009 Cardiac MR Left Ventricle Segmentation Challenge (Radau et al., 2009). The database includes 45 CMR datasets obtained with a $1.5 \mathrm{~T}$ GE Signa MRI, during 10 - to 15 -s breath holds with a temporal resolution of 20 phases over the cardiac cycle. Six to twelve images were obtained from the atrioventricular ring to the apex (thickness $=8 \mathrm{~mm}, F O V=320 \mathrm{~mm} \times 320 \mathrm{~mm}$, matrix $=256 \times 256$ ), with an isotropic pixel spacing ranging from 1.29 to $1.56 \mathrm{~mm}$. The data includes 12 patients with heart failure and myocardial infarction (HF-I), 12 with heart failure but no myocardial infarction (HF-NI), 12 with hypertrophic cardiomyopathy (HYP) and 9 normal cases (N). Moreover, a ground truth, drawn by an experienced cardiologist, is available in all slices for the epicardium and endocardium at the ED phase and for the endocardium at the ES phase. All reported results consider manual contours drawn to include TPMs in the LV cavity.

The segmentation performance was assessed against reference contours using the evaluation code provided in Radau et al. (2009), which evaluates the Dice coefficient, the average perpendicular distance (APD) and the percentage of "good contours" (contours for which the APD is less than $5 \mathrm{~mm}$ ). Moreover, the Hausdorff distance (defined as the maximum perpendicular distance between contours) was also computed to understand the maximum local error of the proposed framework. Each measure was computed slice by slice and a mean value for all slices of a dataset was calculated. However, the original evaluation code only considers "good contours" when computing these means. Besides this limitation, it also removes the slices only present in one of the cardiac phases (ED or ES), disregarding longitudinal contraction. Taking into account the interest in future comparisons with methods that also use this database, the results obtained with the original evaluation code are reported. Nevertheless, to overcome both limitations and fully understand the framework perfor- 

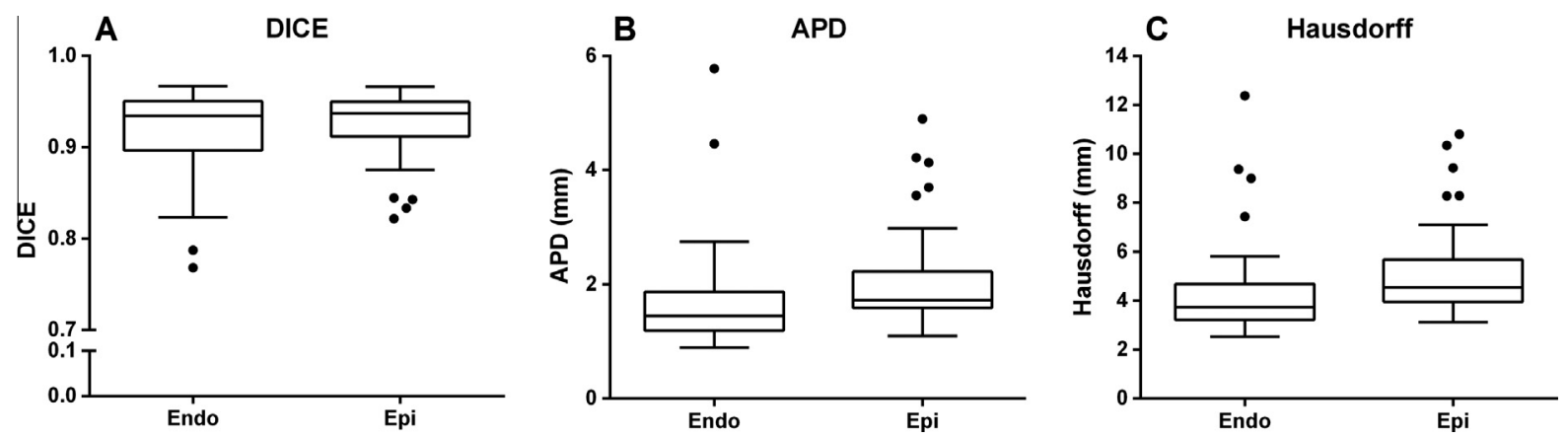

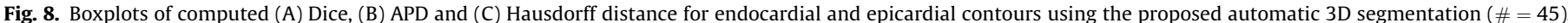

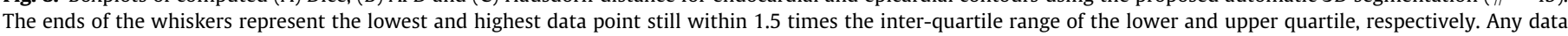
value larger is considered an outlier and plotted as a dot. The results were computed using the customized code.
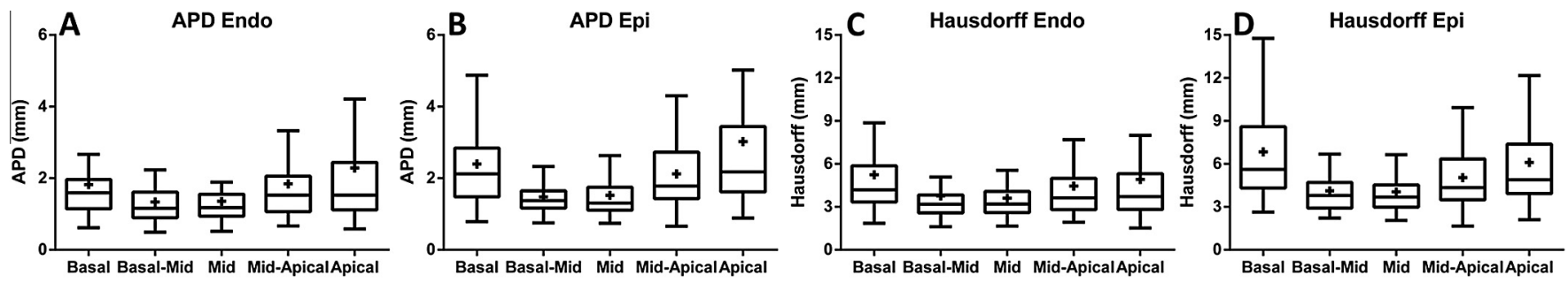

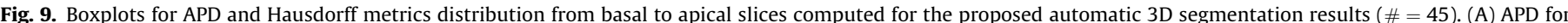

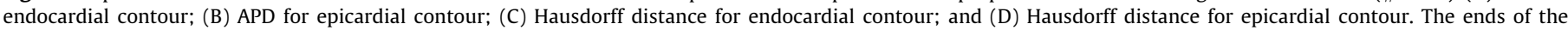

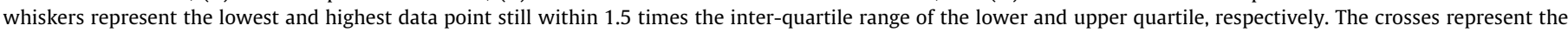
mean values for each metric. The results were computed using the customized code.

mance, the segmentation metrics were also computed considering all the available contours using a customized evaluation code.

The above measures were used to validate both 3D and 3D+time segmentation results. Moreover, our automatic 3D+time framework was compared to the current state-of-the-art methods that used the same database, using the original evaluation code for metrics computation.

In addition to quantitative technical measures, 5 clinical parameters were computed, namely EDV, ESV, SV, LVM and EF, to validate the performance of the proposed 3D+time framework in a clinical context (Petitjean and Dacher, 2011; Hu et al., 2012). Therefore, linear regression and Bland-Altman analysis were performed to assess the correlation between reference clinical indices and the automatically computed ones. Again, the evaluation code presents the aforementioned limitations preventing the correct assessment, but such results are still reported to allow future comparisons for both EF and LVM, as stated in Radau et al. (2009). Nevertheless, the customized code is also used to report clinically correct values using all contours initially considered by the expert during manual segmentation.

Finally, the influence of the parameters variation was assessed to study the robustness and stability of the proposed framework. To this end, the 8 most relevant parameters were included in the analysis, namely the inner and outer region weights ( $w_{\text {in }}$ and $\left.w_{\text {out }}\right)$, the sizes of the local squared regions $\left([n \times n]_{\text {endo }}\right.$ and $\left.[n \times n]_{e p i}\right)$, the threshold values for automatic stack initialization ( $T_{i, \text { endo }}$ and $T_{i, e p i}$ ), the hyperparameter $\alpha$ and the number of points for contour discrete representation $\left(n_{\text {points }}\right)$. The range where the parameter influence is evaluated was defined as a $\pm 50 \%$ variation around the aforementioned chosen parameters values. Each dataset was then segmented using the new value of one parameter while keeping the remaining ones fixed to their original value. Please note that due to the multiscale operation involved in the BEAS framework, $n_{\text {points }}$ should be a base- 2 number. Therefore, its range was defined as a $-50 \%$ to $+100 \%$ variation around the initial predefined value (64 points). Moreover, in the case of the hyperparameter $\alpha$, the zero value was also considered to study the importance of the edge-based term introduced in the 3D epicardial energy functional.

The reported mean computational times were obtained using MATLAB code running on a Intel (R) Core (TM) i5 CPU at $2.4 \mathrm{GHz}$ (shared memory of $4 \mathrm{~GB}$ ).

\section{Results}

Table 1 summarizes the performance for the 3D segmentation, computed using both original evaluation code (OC) and customized code (CC). To also assess the overall performance distribution rather than only average results, Fig. 8 presents the boxplots for each metric for both endocardial and epicardial contours using

Table 1

3D segmentation performance for endocardial and epicardial contours in ED phase $(\#=45, \mu \pm \sigma)$.

\begin{tabular}{|c|c|c|c|c|c|c|c|c|}
\hline & \multicolumn{2}{|l|}{ Dice } & \multicolumn{2}{|l|}{ APD $(\mathrm{mm})$} & \multicolumn{2}{|c|}{ Hausdorff (mm) } & \multicolumn{2}{|c|}{ Good contours (\%) } \\
\hline & Endo & Epi & Endo & Epi & Endo & Epi & Endo & Epi \\
\hline OC & $0.93 \pm 0.03$ & $0.94 \pm 0.02$ & $1.50 \pm 0.47$ & $1.80 \pm 0.41$ & $3.97 \pm 1.24$ & $4.65 \pm 1.14$ & $97.4 \pm 8.2$ & $95.4 \pm 9.6$ \\
\hline $\mathrm{CC}$ & $0.92 \pm 0.04$ & $0.93 \pm 0.03$ & $1.70 \pm 0.89$ & $2.06 \pm 0.84$ & $4.34 \pm 1.91$ & $5.14 \pm 1.79$ & & \\
\hline
\end{tabular}

Original code $(\mathrm{OC})$ only considers "good contours" for metrics computation, while the customized code (CC) uses all segmented slices. 
the customized code. Moreover, the distribution from basal to apical slices of both APD and Hausdorff distance metrics are illustrated in Fig. 9. Note that the Dice metric distribution is not presented, because it is influenced by the relative size of the object (which is smaller in apical slices) and thus not suitable for comparison between slices. Fig. 10 gives three segmentation examples with several slices from base to apex.

Regarding 3D+time LV segmentation, Table 2 presents the overall segmentation plus tracking performance (using CC) for all 45 datasets and also categorized by pathology. Fig. 11 gives an example tracking result over the cardiac cycle.

The comparison of the proposed approach against state-of-theart methods that use the same database is presented in Table 3, using the original evaluation code (Radau et al., 2009) for metrics computation. Moreover, the average total computational time to segment a 4D dataset is also presented.

Regarding the clinical validation, the correlation coefficient obtained by regression analysis and both bias and standard deviation obtained by Bland-Altman analysis are presented in Table 4 for both versions of the evaluation code. Figs. 12 and 13 present the linear regression and Bland-Altman plots, respectively, obtained for EDV, ESV, LVM and EF (using CC).

To better understand the computational burden of each module of the proposed framework, the average computational time for each step is presented in Table 5 along with the average total computational time to segment a dataset.

Finally, Fig. 14 illustrates the influence of the variation of each parameter in the automatic 3D+time segmentation performance. Furthermore, in order to stress the importance of the hybrid energy over a pure region-based one, the anatomical distribution of the epicardial APD errors with and without the proposed edge-based term is presented in Fig. 15.

\section{Discussion}

The first module of the proposed framework was evaluated in the preliminary contribution (Queirós et al., 2013), in which the

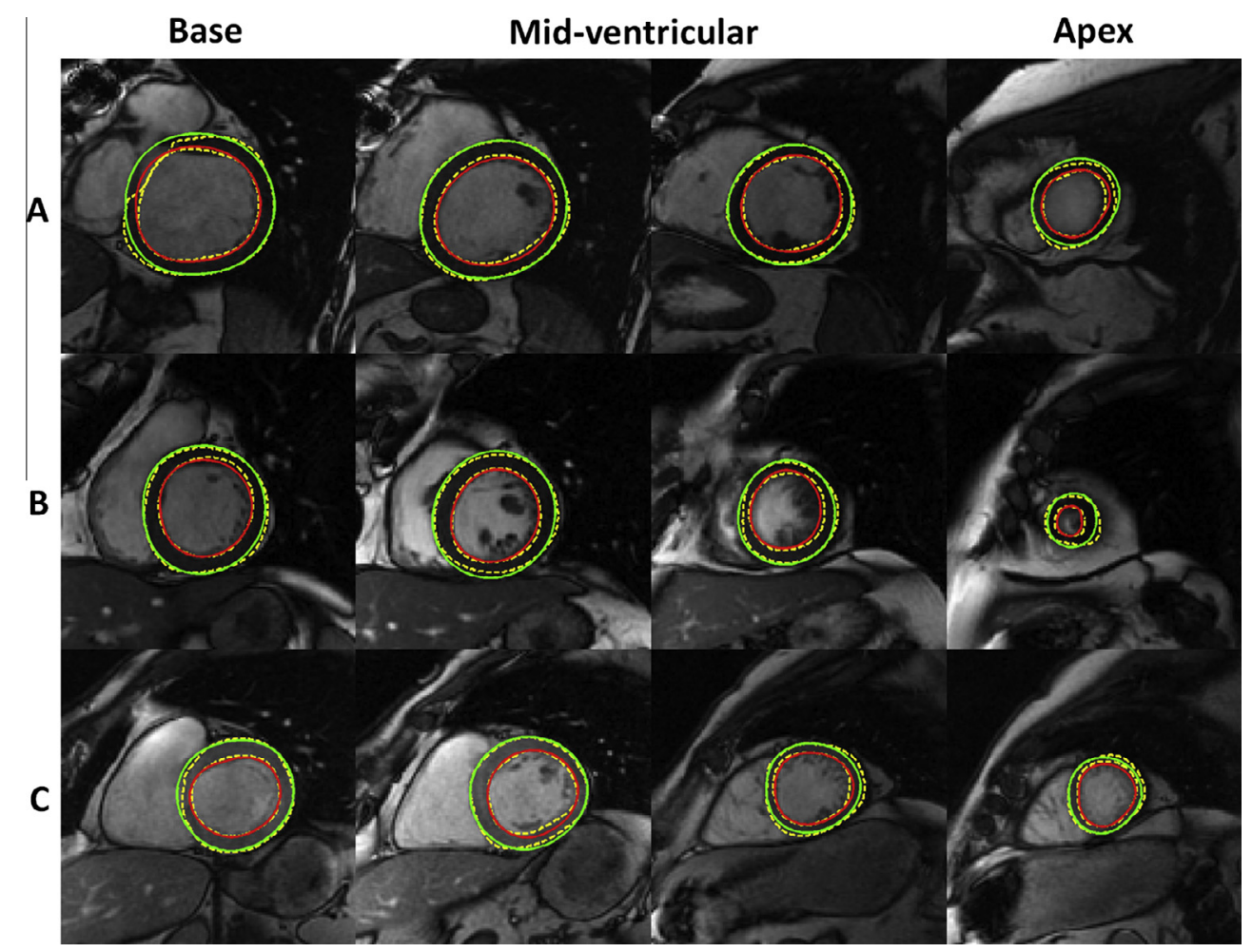

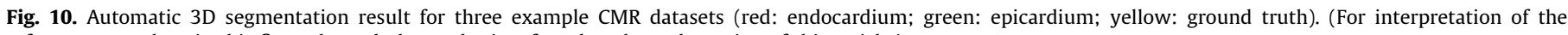
references to colour in this figure legend, the reader is referred to the web version of this article.)

Table 2

3D+time segmentation performance for endocardial and epicardial contours, categorized by pathology and average result (\# - number of datasets, $\mu \pm \sigma$ ).

\begin{tabular}{|c|c|c|c|c|c|c|c|c|c|}
\hline & \multirow[t]{2}{*}{$\#$} & \multicolumn{2}{|l|}{ Dice } & \multicolumn{2}{|l|}{$\mathrm{APD}(\mathrm{mm})$} & \multicolumn{2}{|c|}{ Hausdorff (mm) } & \multicolumn{2}{|c|}{ Good contours (\%) } \\
\hline & & Endo & Epi & Endo & Epi & Endo & Epi & Endo & Epi \\
\hline HF-I & 12 & $0.92 \pm 0.05^{\mathrm{a}, \mathrm{b}}$ & $0.94 \pm 0.03$ & $1.93 \pm 1.28$ & $1.93 \pm 0.96$ & $4.66 \pm 2.38$ & $5.09 \pm 1.91$ & $93.3 \pm 12.0$ & $95.1 \pm 12.6$ \\
\hline HF-NI & 12 & $0.91 \pm 0.03^{\mathrm{c,d}}$ & $0.92 \pm 0.04$ & $2.01 \pm 0.57$ & $2.17 \pm 0.86$ & $5.42 \pm 2.01$ & $5.37 \pm 2.09$ & $94.2 \pm 6.3$ & $93.8 \pm 9.4$ \\
\hline HYP & 12 & $0.81 \pm 0.06^{\mathrm{a}, \mathrm{c}}$ & $0.91 \pm 0.03$ & $2.40 \pm 0.61$ & $2.14 \pm 0.63$ & $4.95 \pm 0.93$ & $5.18 \pm 1.36$ & $90.1 \pm 9.6$ & $98.3 \pm 3.7$ \\
\hline $\mathrm{N}$ & 9 & $0.85 \pm 0.05^{\mathrm{b}, \mathrm{d}}$ & $0.92 \pm 0.03$ & $2.25 \pm 0.79$ & $2.01 \pm 0.83$ & $5.22 \pm 1.58$ & $4.87 \pm 1.65$ & $93.2 \pm 8.6$ & $94.0 \pm 9.9$ \\
\hline Total & 45 & $0.87 \pm 0.07$ & $0.93 \pm 0.03$ & $2.14 \pm 0.88$ & $2.06 \pm 0.84$ & $5.05 \pm 1.85$ & $5.14 \pm 1.79$ & $92.7 \pm 9.5$ & $95.4 \pm 9.6$ \\
\hline
\end{tabular}

Metrics computed using our customized code, which considers all available contours.

a $p<0.05$, unpaired $t$-test between HF-I and HYP.

b $p<0.05$, unpaired $t$-test between HF-I and N.

c $p<0.05$, unpaired $t$-test between HF-NI and HYP.

d $p<0.05$, unpaired $t$-test between HF-NI and N. 


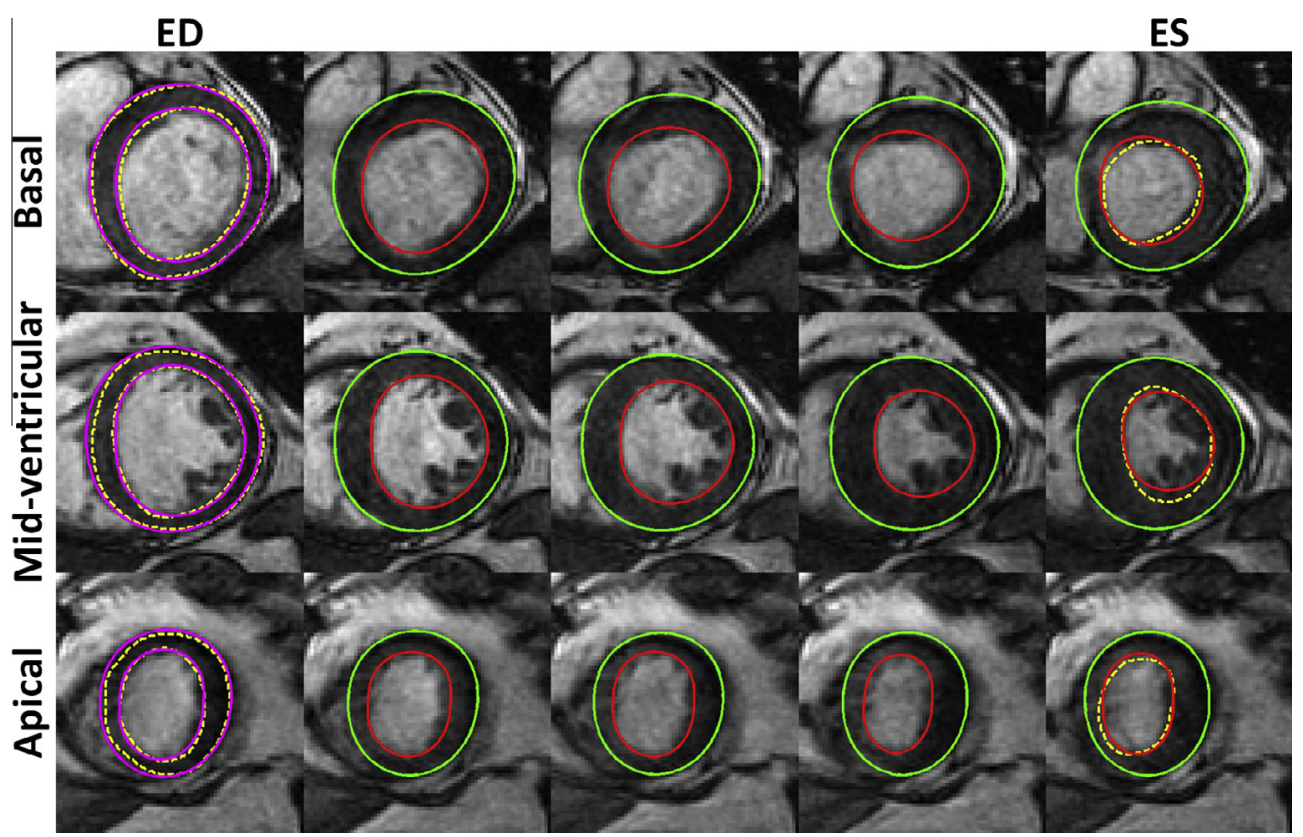

Fig. 11. Automatic 3D+time segmentation result for an example CMR dataset (magenta: automatic contour at ED phase; red: endocardium; green: epicardium; yellow: ground truth). (For interpretation of the references to colour in this figure legend, the reader is referred to the web version of this article.)
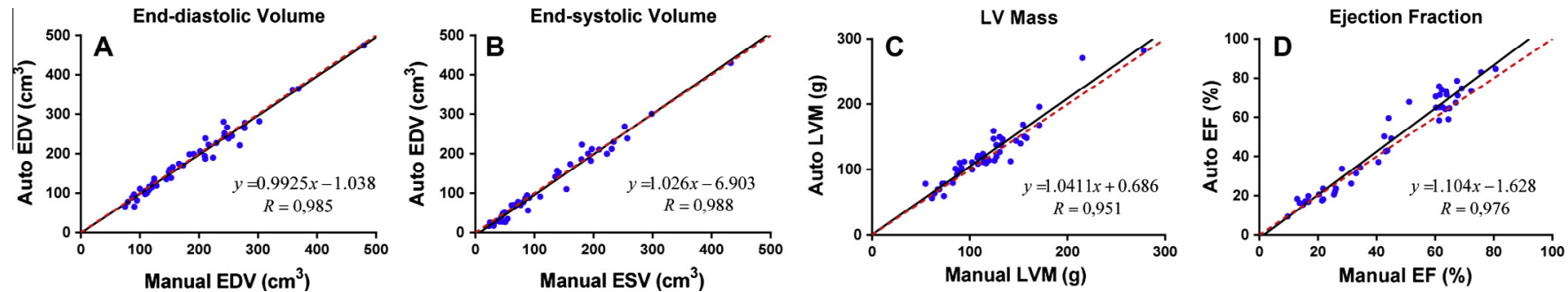

Fig. 12. Linear regression for (A) end-diastolic volume (EDV); (B) end-systolic volume (ESV); (C) LV mass (LVM); and (D) and ejection fraction (EF).

Table 3

Comparison of segmentation performance between proposed approach and state-of-the-art methods (\# - number of datasets evaluated, $\mu \pm \sigma$ ).

\begin{tabular}{|c|c|c|c|c|c|c|c|c|}
\hline \multirow[t]{2}{*}{ Method } & \multirow[t]{2}{*}{$\#$} & \multirow[t]{2}{*}{ Time (s) } & \multicolumn{2}{|l|}{ Dice } & \multicolumn{2}{|l|}{ APD (mm) } & \multicolumn{2}{|c|}{ Good contours (\%) } \\
\hline & & & Endo & Epi & Endo & Epi & Endo & Epi \\
\hline Proposed & 45 & $11.13 \pm 2.76$ & $0.90 \pm 0.05$ & $0.94 \pm 0.02$ & $1.76 \pm 0.45$ & $1.80 \pm 0.41$ & $92.7 \pm 9.5$ & $95.4 \pm 9.6$ \\
\hline Constantinides et al. (2009) & 30 & - & $0.89 \pm 0.04$ & $0.92 \pm 0.02$ & $2.04 \pm 0.47$ & $2.35 \pm 0.57$ & 90.35 & 92.56 \\
\hline Constantinides et al. (2012) & 45 & $\sim 60$ & $0.86 \pm 0.05$ & $0.91 \pm 0.03$ & $2.44 \pm 0.56$ & $2.80 \pm 0.71$ & $80 \pm 16$ & $71 \pm 26$ \\
\hline Hu et al. (2012) & 45 & $153.86 \pm 32.13$ & $0.89 \pm 0.03$ & $0.94 \pm 0.02$ & $2.24 \pm 0.40$ & $2.19 \pm 0.49$ & $91.06 \pm 9.4$ & $91.21 \pm 8.5$ \\
\hline Huang et al. (2011) & 45 & - & $0.89 \pm 0.04$ & $0.93 \pm 0.02$ & $2.16 \pm 0.46$ & $2.22 \pm 0.43$ & $79.2 \pm 19.0$ & $83.9 \pm 16.8$ \\
\hline Jolly (2009) & 30 & $\sim 60$ & $0.88 \pm 0.04$ & $0.93 \pm 0.02$ & $2.26 \pm 0.59$ & $1.97 \pm 0.48$ & $95.62 \pm 8.8$ & $97.29 \pm 5.8$ \\
\hline Liu et al. (2012) & 45 & $129.45 \pm 30.39$ & $0.88 \pm 0.03$ & $0.94 \pm 0.02$ & $2.36 \pm 0.39$ & $2.19 \pm 0.49$ & $91.17 \pm 8.5$ & $90.78 \pm 10.7$ \\
\hline Schaerer et al. (2010) & 45 & - & $0.87 \pm 0.04$ & $0.92 \pm 0.02$ & $2.97 \pm 0.38$ & $3.14 \pm 0.33$ & - & - \\
\hline Uzunbas et al. (2012) & 15 & $\sim 45$ & $0.82 \pm 0.06$ & $0.91 \pm 0.03$ & $2.98 \pm 0.88$ & $1.78 \pm 0.35$ & - & - \\
\hline Wijnhout et al. (2009) & 15 & $\sim 60$ & $0.89 \pm 0.03$ & $0.93 \pm 0.01$ & $2.29 \pm 0.57$ & $2.28 \pm 0.39$ & $86.4 \pm 11.0$ & $94.2 \pm 7.0$ \\
\hline
\end{tabular}

Metrics computed using original evaluation code, which considers only "good contours" for metrics computation.

Table 4

Linear regression and Bland-Altman analysis for clinical cardiac indexes ( $\#=45$ ).

\begin{tabular}{|c|c|c|c|c|c|c|c|c|c|c|c|c|c|c|c|}
\hline & \multicolumn{3}{|l|}{ EDV } & \multicolumn{3}{|l|}{ ESV } & \multicolumn{3}{|l|}{ SV } & \multicolumn{3}{|l|}{ LVM } & \multicolumn{3}{|l|}{$\mathrm{EF}$} \\
\hline & $\mathrm{R}$ & Bias & $\sigma$ & $\mathrm{R}$ & Bias & $\sigma$ & $\mathrm{R}$ & Bias & $\sigma$ & $\mathrm{R}$ & Bias & $\sigma$ & $\mathrm{R}$ & Bias & $\sigma$ \\
\hline OC & - & - & - & - & - & - & - & - & - & 0.906 & -0.01 & 18.24 & 0.977 & $2.45^{\mathrm{a}}$ & 4.83 \\
\hline $\mathrm{CC}$ & 0.985 & -2.46 & 15.03 & 0.988 & -3.83 & 14.78 & 0.955 & 1.38 & 8.07 & 0.951 & $5.51^{\mathrm{a}}$ & 14.13 & 0.976 & $3.00^{\mathrm{a}}$ & 5.60 \\
\hline
\end{tabular}

Original code (OC) only considers "good contours" for metrics computation, while the customized code (CC) uses all segmented slices.

$\mathrm{R}$ is the Pearson product-moment correlation coefficient

a $p<0.05$, paired $t$-test against zero. 

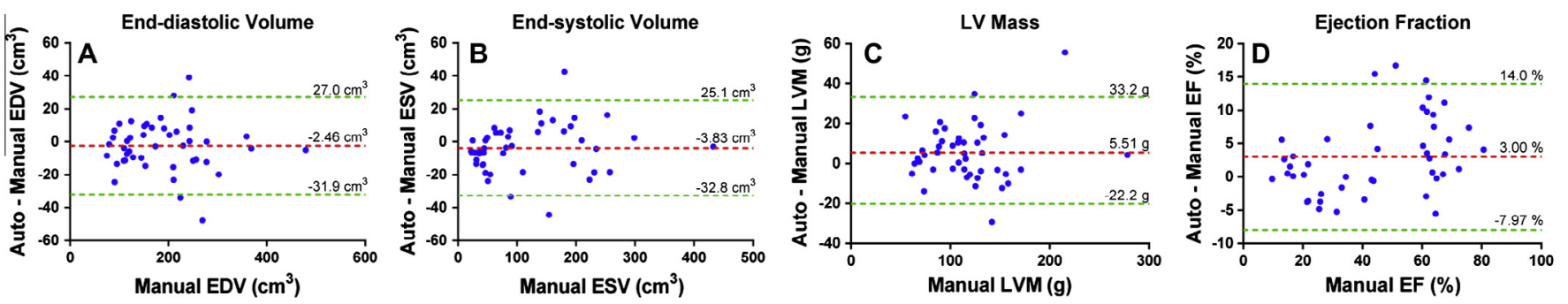

Fig. 13. Bland-Altman analysis for (A) end-diastolic volume (EDV); (B) end-systolic volume (ESV); (C) LV mass (LVM); and (D) and ejection fraction (EF).

Table 5

Average computational time for each step of the proposed approach ( $\#=45, \mu \pm \sigma$ ).

\begin{tabular}{lr}
\hline Method & \multicolumn{1}{c}{ Time $(\mathrm{s})$} \\
\hline LV location & $0.10 \pm 0.02$ \\
Template matching & $1.16 \pm 0.37$ \\
2D segmentation & $0.22 \pm 0.05$ \\
Stack initialization & $0.55 \pm 0.10$ \\
3D segmentation & $6.21 \pm 2.41$ \\
Anatomical affine optical flow & $2.89 \pm 0.49$ \\
Total & $11.13 \pm 2.76$ \\
\hline
\end{tabular}

2D automatic initialization algorithm showed a high feasibility (91.1\%) and robustness. However, four wrongly initialized images were observed in the 45 datasets used (Queirós et al., 2013). Nonetheless, we still included these datasets in the present 3D and $3 \mathrm{D}+$ time analysis, as it was found that the proposed stack initialization is able to recover from these bad initializations. Regarding the second module, the proposed energy for 2D segmentation combined with the automatic 2D initialization resulted in an accurate endo and epicardial delineations (data not shown). Hence, the first block of the proposed framework showed to be efficient and robust to obtain a first mid-ventricular delineation for further initialization.

Concerning the proposed automatic stack initialization, the ability to include the 4 wrongly initialized images may be linked to the robustness of the EM algorithm and the simple but powerful principle of thresholding. In other words, even if the initial 2D midventricular segmentation has a sub-optimal result, the applied mask and subsequent EM algorithm still allows a good estimation of the intensity distribution of the LV cavity and myocardial wall. After properly estimating the two gaussian distributions, both myocardial and cavity thresholds are also appropriately defined. Consequently, the initial contours obtained for all slices from base to apex are fairly close to the true boundaries, which permits the proposed 3D localized energy functional to obtain an accurate result during segmentation. In fact, 2 of these 4 cases where the 2D initialization failed ended up having a 3D segmentation with errors below average. Moreover, the proposed method is at the same time able to adequately estimate and reduce occasional misalignment between slices. Such feature is possible due to the center estimation during contour growing, which adapts itself to dataset-specific acquisition displacement artifacts (Fig. 6). Although such strategy might not be anatomically correct, as it could overcompensate for ventricles presenting curvilinear longaxis (e.g., banana-shaped heart) and thus deviate from the physiologic LV axis, it allows for a 3D spatial continuous delineation when using the proposed cylindrical topology.

Please note that the proposed framework assumes that the stack of slices was previously cropped to only include slices covering the LV in the stack initialization and subsequent segmentation. In case this was not done, a simple solution would be to have the user input two points at run-time, corresponding to the choice of basal and apical slices. Although the presented framework does not require any user interaction during the segmentation itself, such pre-processing of the data is still required. In order to obtain a fully automatic methodology, we intend to address this issue in future work.

Regarding Table 1, the proposed 3D segmentation method and associated hybrid energy functional proved to give accurate results in all metrics used. A high percentage of "good contours" was obtained, with an average of $97.4 \%$ and $95.4 \%$ for endo and epicardial contours, respectively. The slices considered bad $(A P D \geqslant 5 \mathrm{~mm}$ ) were mostly located in the apex (due to small size and low contrast with respect to the background) or in basal slices in the presence of the left ventricular outflow tract (LVOT), as illustrated in Fig. 9. The latter problem (i.e., myocardium appearing as an incomplete annular shape) was still not directly addressed in the proposed framework, being only avoided in most cases due to the intra- and inter-slice spatial continuity of the LV surface (see Fig. 10A, at the base). Nevertheless, it increases the measured distances and is the main contributor to the reported average Hausdorff distance. Note the increased average and standard deviation in this metric when considering all slices (CC compared to OC), although only a few percentage of contours are added. As expected, the reported values for our customized code are lower due to the inclusion of slices considered poorly segmented. Such results reinforce the need to look together to both metrics and the percentage of "good contours" when using the original evaluation code.

Fig. 8 gives an overview of the proposed methodology performance in segmenting the ED phase, with more than $75 \%$ of the studied datasets having a Dice metric higher than 0.90 for both endo and epicardial contours. At the same time, these datasets also present an APD under $2 \mathrm{~mm}$ for both contours and a maximum error around $4-5 \mathrm{~mm}$. Taking into account the observed outliers, it is important to note that two of these are from the 4 cases having a wrong 2D initialization. The other outliers are associated with datasets with considerable heterogeneity in blood image intensity between slices, hampering the correct automatic initialization of apical and basal slices further away from the initial mid-ventricular slice when using our threshold-based strategy. Nonetheless, despite its low-level intensity assumptions, the proposed method proved to be globally generic.

Regarding the error distribution from basal to apical slices in Fig. 9, higher errors are observed for the two ends of the left ventricle, mainly due to the LVOT in basal slices and to PVE in apical ones (as stated above). Notwithstanding, overall the segmentation is robust independently from the slice level. Fig. 10 confirms the robustness of the proposed 3D segmentation against different myocardial intensities, contrast and presence of TPMs (note the third column of Fig. 10B).

The same robustness is observed in the 3D+time approach (Table 2). However, a decrease in the endocardium accuracy for the 3 measured metrics is detected compared to the above 3D results (Table 1 ). This is partially related with the greater difficulty in distinguishing the compacted TPMs from the myocardial tissue in ES slices, hampering the correct identification of the endocardial 

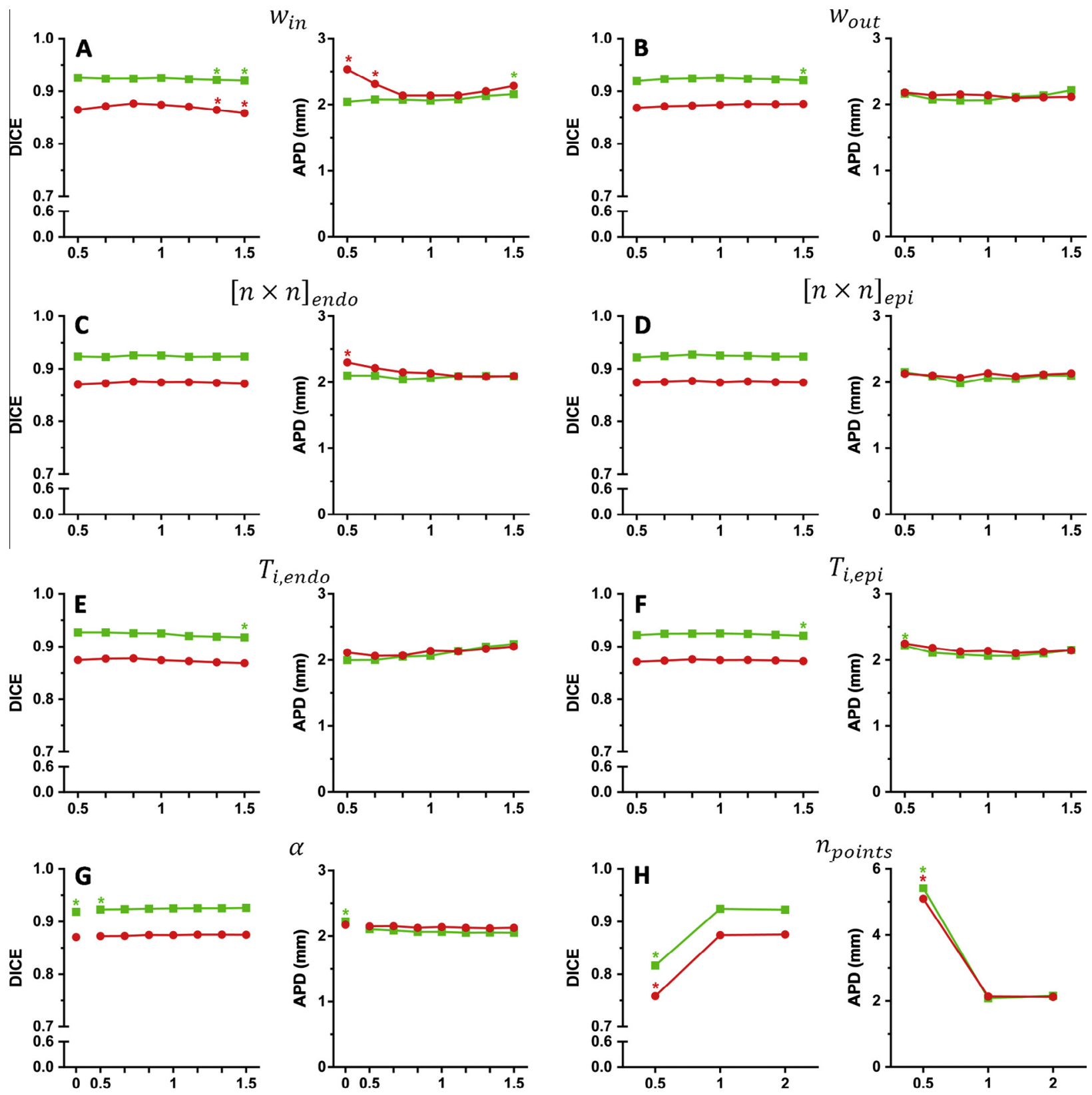

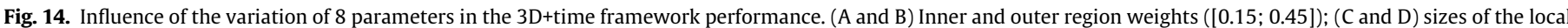

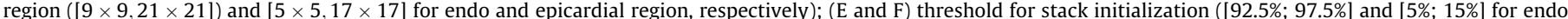

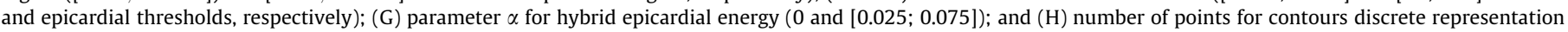

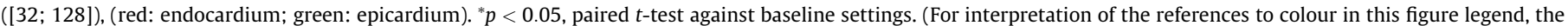
reader is referred to the web version of this article.)

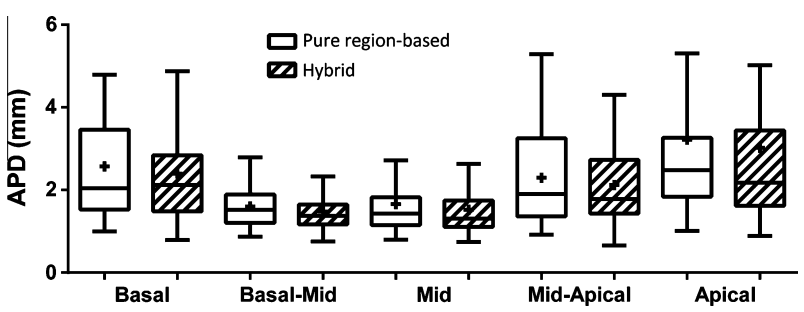

Fig. 15. Boxplots of epicardial APD distribution from basal to apical slices computed using a pure region-based epicardial energy and a hybrid energy functional. The ends of the whiskers represent the lowest and highest data point still within 1.5 times the inter-quartile range of the lower and upper quartile, respectively. The crosses represent the mean values for each metric. The results were computed using the customized code. boundary. This, in combination with the LVOT in basal slices and PVE in apical ones, as already discussed for the ED frame, is the main cause for contours considered badly segmented in the ES frame. Nevertheless, the percentage of "good contours" is still high for the endocardium, with an average $92.3 \%$. Moreover, the APD is kept around $2 \mathrm{~mm}$ when considering all contours in both ED and ES phases. Note that epicardial results are unchanged as they are not evaluated in ES phase due to lack of manual contouring. When dividing the studied datasets by pathology, no significant difference is found between average APD between these groups. Regarding the Dice metric, significant differences were found for the endocardial contour between some groups (Table 2). However, this can be related to the different cavity size between groups of subjects, since the differences in average APD are not significant. In 
summary, these results suggest that the proposed algorithm is robust against different pathologies and ventricular anatomy and function. At the same time, it corroborates the feasibility of using an affine transform to describe the in-plane LV motion during the 3D+time tracking stage of the proposed algorithm.

According to Table 3, the proposed framework proved to be competitive, as compared to the state-of-the-art frameworks that used this database for validation, providing leading results for both accuracy and computational times. This observation was valid for all segmentation performance measures employed, with a smaller average APD, superior average Dice metric and one of the highest percentage of "good contours". Note that the original evaluation code only considers "good contours" for computation of means, implying that our results are more accurate even considering a higher percentage of slices. On the other hand, it is important to note that the results for some of the state-of-the-art methods were obtained during the MICCAI challenge, therefore being tested in one set and validated in another. In our case, we tested and validated on the full database. Nevertheless, the presented sensitivity analysis (Fig. 14) proved the robustness of the algorithm, discrediting the possibility of over-tuning of the different parameters of the proposed algorithm. Finally, besides presenting high accuracy, the framework showed also to be very fast (Table 5), presenting an average total computational time of $11.16 \pm 2.76 \mathrm{~s}$ per dataset (around $0.06-0.09 \mathrm{~s}$ per image) in a Matlab implementation. Since a fully manual segmentation takes between 6 to 20 min for only ED and ES phases (Hautvast et al., 2012; Petitjean and Dacher, 2011), the proposed framework seems to be fast and accurate enough to be of interest in daily clinical practice.

Linear regression revealed a high correlation for all clinical cardiac indices, ultimately showing the accuracy and interest of the proposed framework for automatic assessment of LV morphology and global function (Table 4 and Fig. 12). Regarding Bland-Altman limits of agreement (Fig. 13), the reported values are slightly higher than other frameworks in the literature (Eslami et al., 2013; Cordero-Grande et al., 2011). Nonetheless, these methods require some user interaction or were only validated on patients with a specific pathology. In the present work, an automatic framework is being proposed and validated on a database with both healthy subjects and patients.

Notably, the sensitivity analysis of the proposed framework (Fig. 14) reveals that the overall performance is not significantly impaired within a fairly large range of parameter settings. The most critical choice for the overall segmentation performance was found to be the number of points used to represent the myocardial contours (Fig. 14H). However, using 32 points instead of the proposed 64 points corresponds to a quite severe downsampling of the surface representation, which strongly constrains the degrees of freedom of the segmented LV shape. On the other hand, using 128 points to represent the contours did not lead to any significant accuracy improvement, thus reflecting that the proposed 64 points provides an optimal balance between overall segmentation accuracy and robustness, as well as reduced computational burden. The influence of the endocardial scalar weight $w_{i n}$ was found to also have a statistically significant influence on the segmentation performance for variations of $\pm 30 \%$ (Fig. 14A). This is intrinsically linked to the fact that this term controls the equilibrium point between the region forces from the blood pool and myocardium, thus being translated as the amount of trabeculae included in the myocardial region. On the other hand, the influence of the epicardial scalar weight $w_{\text {out }}$ did not have such variability pattern, statistically showing significant influence only on the Dice metric for a variation of $+50 \%$ (Fig. 14B). All the other parameters either did not show any statistically significant influence on the segmentation performance or only showed small but significant effects for variations of $\pm 50 \%$.
Additionally, in the case of a pure localized region-based energy for the epicardial contour in the 3D segmentation $(\alpha=0)$, there was a small yet statistically significant decrease on the global performance, mostly perceived in the apical slices (see Fig. 15). Such result is primarily linked to the increased capture range of the edge-based term, which helps handling the blurry appearance of the myocardium in apical slices (caused by partial volume effects). A similar effect is achieved in cases of slightly worst initializations, where the edge-based term counteracts the increased sensitivity to the initial position of the region-based term. Overall, this result reinforces the interest of the proposed hybrid energy functional in the $3 \mathrm{D}$ segmentation.

A note should be addressed to the proposed regularization terms. Whereas the minimum thickness penalty was found to have an important contribution by preventing the local overlap of the endocardial and epicardial interfaces, the proposed curvature regularization approach, included to deal with concave endocardial regions, was found to have a small segmentation error reduction ( $<5 \%$ in all metrics). However, the segmentation results became visually more appealing, while it also positively contributed in more challenging slices. Thus, this term was kept in the 3D segmentation step of the proposed framework. Note that the curvature-based prior was only included in this step, since the 2D case is simply used as an initialization step towards the subsequent 3D segmentation. In fact, and as stated above, the automatic stack initialization step is capable of recovering in cases of sub-optimal 2D segmentation results, thus avoiding the need for such extra 2D regularization term.

Overall, the present analysis showed the robustness of the proposed framework, with only slight variations in the segmentation performance for most of the parameters analyzed. This result is of utmost importance to demonstrate its feasibility against variability, highlighting the robustness of the method.

\section{Conclusion}

In summary, a novel automatic 3D+time segmentation framework for CMR datasets was presented. The proposed approach was shown to be efficient, accurate, robust and fast compared to the other state-of-the-art methods, presenting leading results in both accuracy and computational time in the common database used for comparative purposes. The computational time to analyze an entire 4D dataset took on average $11 \mathrm{~s}$, with an overall correlation with reference contours of $0.99,0.99,0.96,0.95$ and 0.98 for EDV, ESV, SV, LVM and EF, respectively. Such results show the appeal of the proposed framework for automatic assessment of LV morphology and global function in daily clinical practice.

In the future, the proposed algorithm should be prospectively evaluated in a larger database comprehending "real-world" variability in image quality and anatomical and functional characteristics.

\section{Appendix A. Coupled BEAS energy derivation}

We consider here the differentiation of the coupled localized region-based energy functional, $E(7)$, with respect to a given Bspline coefficient $c\left[\mathbf{k}_{\mathbf{j}}\right]$. Given (7), one can rewrite it as:

$$
E=\sum_{i=1}^{2} \int_{\Omega} \delta_{\phi_{i}}(\mathbf{x}) \int_{\Omega} B(\mathbf{x}, \mathbf{y}) \cdot F_{i}(\mathbf{y}) d \mathbf{y} d \mathbf{x}=\sum_{i=1}^{2} E_{i},
$$

with $i=1$ and $i=2$ representing the endocardium and epicardium, respectively,

Now, let consider the derivation of $E_{i}$ only: 
$\frac{\partial E_{i}}{\partial c_{W_{p}}\left[\mathbf{k}_{\mathbf{j}}\right]}=\int_{\Omega} \delta_{\phi_{i}}(\mathbf{x}) \int_{\Omega} B(\mathbf{x}, \mathbf{y}) \frac{\partial F_{i}}{\partial \phi_{i}} \cdot \frac{\partial \phi_{i}}{\partial c_{W_{P}}\left[\mathbf{k}_{\mathbf{j}}\right]} d \mathbf{y} d \mathbf{x}$

The term $\frac{\partial F_{i}}{\partial \phi_{i}}$ depends only on the feature function used and can be expressed as (Barbosa et al., 2012; Barbosa et al., 2013a):

$\frac{\partial F_{i}(\mathbf{y})}{\partial \phi_{i}}=g_{i}(\mathbf{y}) \cdot \delta_{\phi_{i}}(\mathbf{y})$

Noting moreover that $B(\mathbf{x}, \mathbf{y}) \delta_{\phi}(\mathbf{x})$ is different from zero only if $\mathbf{x}=\mathbf{y}$, we have $B(\mathbf{x}, \mathbf{y}) \delta_{\phi}(\mathbf{x})=\delta_{\phi}(\mathbf{x}-\mathbf{y})$ and therefore,

$\frac{\partial E_{i}}{\partial c_{W_{P}}\left[\mathbf{k}_{\mathbf{j}}\right]}=\int_{\Omega} g_{i}(\mathbf{x}) \frac{\partial \phi_{i}(\mathbf{x})}{\partial c_{W_{P}}\left[\mathbf{k}_{\mathbf{j}}\right]} \delta_{\phi_{i}}(\mathbf{x}) d \mathbf{x}$

Moreover, we have:

$\phi_{\text {endo }}(\mathbf{x})=\Gamma_{\text {endo }}\left(\mathbf{x}^{*}\right)-x_{1}=\psi_{W_{P}}\left(\mathbf{x}^{*}\right)-\psi_{W_{T}}\left(\mathbf{x}^{*}\right)-x_{1}$

and

$\phi_{e p i}(\mathbf{x})=\Gamma_{e p i}\left(\mathbf{x}^{*}\right)-x_{1}=\psi_{W_{P}}\left(\mathbf{x}^{*}\right)+\psi_{W_{T}}\left(\mathbf{x}^{*}\right)-x_{1}$,

which, from (1), leads to:

$\frac{\partial \phi_{\text {endo }}(\mathbf{x})}{\partial c_{W_{P}}\left[\mathbf{k}_{\mathbf{j}}\right]}=\beta^{d}\left(\frac{\mathbf{x}^{*}}{h}-\mathbf{k}_{\mathbf{j}}\right)$

and

$\frac{\partial \phi_{e p i}(\mathbf{x})}{\partial c_{W_{P}}\left[\mathbf{k}_{\mathbf{j}}\right]}=\beta^{d}\left(\frac{\mathbf{x}^{*}}{h}-\mathbf{k}_{\mathbf{j}}\right)$

Eq. (A.4) thus becomes:

$\frac{\partial E_{i}}{\partial c_{W_{P}}\left[\mathbf{k}_{\mathbf{j}}\right]}=\int_{\Omega} g_{i}(\mathbf{x}) \beta^{d}\left(\frac{\mathbf{x}^{*}}{h}-\mathbf{k}_{\mathbf{j}}\right) \delta_{\phi_{i}}(\mathbf{x}) d \mathbf{x}$

Taking into account the following property (Barbosa et al., 2012):

$\int_{\Omega} f(\mathbf{x}) \delta_{\phi}(\mathbf{x}) d \mathbf{x}=\int_{\Gamma} \bar{f}\left(\mathbf{x}^{*}\right) d \mathbf{x}^{*}$,

we get:

$\frac{\partial E_{i}}{\partial c_{W_{P}}\left[\mathbf{k}_{\mathbf{j}}\right]}=\int_{\Gamma_{i}} \bar{g}_{i}\left(\mathbf{x}^{*}\right) \beta^{d}\left(\frac{\mathbf{x}^{*}}{h}-\mathbf{k}_{\mathbf{j}}\right) d \mathbf{x}^{*}$

and, thus, yielding Eq. (12). Using the same reasoning, we can get Eq. (13).

\section{References}

Anderson, J., Weaver, A., Horne, B., Jones, H., Jelaco, G., Cha, J., Busto, H., Hall, J., Walker, K., Blatter, D., 2007. Normal cardiac magnetic resonance measurements and interobserver discrepancies in volumes and mass using the papillary muscle inclusion method. Open Gen. Internal Med. J. 1, 6-12.

Barbosa, D., Bernard, O., Savu, O., Dietenbeck, T., Heyde, B., Claus, P., Friboulet, D., D'hooge, J., 2010. Coupled B-spline active geometric functions for myocardial segmentation: a localized region-based approach. In: IEEE International Ultrasonics Symposium (IUS), pp. 1648-1651.

Barbosa, D., Dietenbeck, T., Schaerer, J., D’hooge, J., Friboulet, D., Bernard, O., 2012. B-spline explicit active surfaces: an efficient framework for real-time 3-D region-based segmentation. IEEE Trans. Image Process. 21, 241-251.

Barbosa, D., Dietenbeck, T., Heyde, B., Houle, H., Friboulet, D., D’hooge, J., Bernard, O., 2013a. Fast and fully automatic 3-D echocardiographic segmentation using bspline explicit active surfaces: feasibility study and validation in a clinical setting. Ultrasound Med. Biol. 39, 89-101.

Barbosa, D., Heyde, B., Dietenbeck, T., Friboulet, D., D’hooge, J., Bernard, O., 2013b. Fast left ventricle tracking in 3d echocardiographic data using anatomical affine optical flow. In: Proceedings of Functional Imaging and Modeling of the Heart (FIMH2013), pp. 191-199.

Ben Ayed, I., Li, S., Ross, I., 2009. Embedding overlap priors in variational left ventricle tracking. IEEE Trans. Med. Imag. 28, 1902-1913.

Ben Ayed, I., Chen, H., Punithakumar, K., Ross, I., Li, S., 2012. Max-flow segmentation of the left ventricle by recovering subject-specific distributions via a bound of the Bhattacharyya measure. Med. Image Anal. 16, 87-100.
Canny, J., 1986. A computational approach to edge detection. IEEE Trans. Pattern Anal. Mach. Intell., 679-698.

Chan, T., Vese, L., 2001. Active contours without edges. IEEE Trans. Image Process. $10,266-277$.

Chen, T. Babb, J., Kellman, P. Axel, L, Kim, D., 2008. Semiautomated segmentation of myocardial contours for fast strain analysis in cine displacement-encoded MRI. IEEE Trans. Med. Imag. 27, 1084-1094.

Ciofolo, C., Fradkin, M., Mory, B., Hautvast, G., Breeuwer, M., 2008. Automatic myocardium segmentation in late-enhancement MRI. In: IEEE International Symposium on Biomedical Imaging (ISBI), pp. 225-228.

Constantinides, C., Chenoune, Y., Kachenoura, N., Roullot, E., Mousseaux, E. Herment, A., Frouin, F., 2009. Semi-automated cardiac segmentation on cine magnetic resonance images using GVF-Snake deformable models. MIDAS J. Card. MR Left Ventricle Segmentation Challenge.

Constantinides, C., Roullot, E., Lefort, M., Frouin, F., 2012. Fully automated segmentation of the left ventricle applied to cine MR images: description and results on a database of 45 subjects. In: IEEE Engineering in Medicine and Biology Society (EMBC). IEEE, pp. 3207-3210.

Cordero-Grande, L, Vegas-Sánchez-Ferrero, G, Casaseca-de-la Higuera, P. Alberto San-Román-Calvar, J., Revilla-Orodea, A., Martín-Fernández, M., Alberola-López, C., 2011. Unsupervised 4d myocardium segmentation with a markov random field based deformable model. Med. Image Anal. 15, 283-301.

Dietenbeck, T., Alessandrini, M., Barbosa, D., D’hooge, J., Friboulet, D., Bernard, O. 2011. Detection of the whole myocardium in 2D-echocardiography for multiple orientations using a geometrically constrained level-set. Med. Image Anal.

Eslami, A., Karamalis, A., Katouzian, A., Navab, N., 2013. Segmentation by retrieval with guided random walks: application to left ventricle segmentation in MRI. Med. Image Anal..

Hautvast, G., Lobregt, S., Breeuwer, M., Gerritsen, F., 2006. Automatic contour propagation in cine cardiac magnetic resonance images. IEEE Trans. Med. Imag. $25,1472-1482$.

Hautvast, G., Salton, C., Chuang, M., Breeuwer, M., O’Donnell, C., Manning, W., 2012. Accurate computer-aided quantification of left ventricular parameters: experience in 1555 cardiac magnetic resonance studies from the Framingham heart study. Mag. Reson. Med..

Huang, S., Liu, J., Lee, L., Venkatesh, S., Teo, L., Au, C., Nowinski, W., 2011. An imagebased comprehensive approach for automatic segmentation of left ventricle from cardiac short axis cine MR images. J. Digit. Imag. 24, 598-608.

Hu, H., Liu, H., Gao, Z., Huang, L., 2012. Hybrid segmentation of left ventricle in cardiac MRI using Gaussian-mixture model and region restricted dynamic programming. Mag. Reson. Imag.

Jolly, M., 2009. Fully automatic left ventricle segmentation in cardiac cine MR images using registration and minimum surfaces. MIDAS J. - Card. MR Left Ventricle Segmentation Challenge.

Lankton, S., Tannenbaum, A., 2008. Localizing region-based active contours. IEEE Trans. Image Process. 17, 2029-2039.

Lee, H., Codella, N., Cham, M., Weinsaft, J., Wang, Y., 2010. Automatic left ventricle segmentation using iterative thresholding and an active contour model with adaptation on short-axis cardiac MRI. IEEE Trans. Biomed. Eng. 57, 905-913.

Lin, N., Yu, W., Duncan, J.S., 2003. Combinative multi-scale level set framework for echocardiographic image segmentation. Med. Image Anal. 7, 529-537.

Liu, H., Hu, H., Xu, X., Song, E., 2012. Automatic left ventricle segmentation in cardiac MRI using topological stable-state thresholding and region restricted dynamic programming. Acad. Radiol..

Lu, Y., Radau, P., Connelly, K., Dick, A., Wright, G., 2009. Segmentation of left ventricle in cardiac cine MRI: an automatic image-driven method. Funct. Imag. Model. Heart, 339-347.

Lynch, M., Ghita, O., Whelan, P., 2008. Segmentation of the left ventricle of the heart in $3 \mathrm{D}+\mathrm{t}$ MRI data using an optimized nonrigid temporal model. IEEE Trans. Med. Imag. 27, 195-203.

Miller, C., Pearce, K., Jordan, P., Argyle, R., Clark, D., Stout, M., Ray, S., Schmitt, M., 2012a. Comparison of real-time three-dimensional echocardiography with cardiovascular magnetic resonance for left ventricular volumetric assessment in unselected patients. Eur. Heart J. 13, 187-195.

Miller, C.A., Jordan, P., Borg, A., Argyle, R., Clark, D., Pearce, K., Schmitt, M., 2012b. Quantification of left ventricular indices from SSFP cine imaging: impact of realworld variability in analysis methodology and utility of geometric modeling. J. Mag. Reson. Imag..

Moon, T., 1996. The expectation-maximization algorithm. Signal Process. Mag., IEEE $13,47-60$.

Otsu, N., 1975. A threshold selection method from gray-level histograms. Automatica 11, 23-27.

Paragios, N., 2002. A variational approach for the segmentation of the left ventricle in cardiac image analysis. Int. J. Comput. Vis. 50, 345-362.

Pednekar, A., Kurkure, U., Muthupillai, R., Flamm, S., Kakadiaris, I.A. 2006. Automated left ventricular segmentation in cardiac MRI. IEEE Trans. Biomed. Eng. 53, 1425-1428.

Petitjean, C., Dacher, J., 2011. A review of segmentation methods in short axis cardiac MR images. Med. Image Anal. 15, 169-184.

Pluempitiwiriyawej, C., Moura, J., Wu, Y., Ho, C., 2005. STACS: New active contour scheme for cardiac MR image segmentation. IEEE Trans. Med. Imag. 24, $593-$ 603.

Queirós, S., Barbosa, D., Heyde, B., Morais, P., Friboulet, D., Claus, P., Bernard, O. D'hooge, J., 2013. A fast fully automatic segmentation of the myocardium in $2 d$ 
MR images. In: Proceedings of Functional Imaging and Modeling of the Heart (FIMH2013), pp. 71-79.

Radau, P., Lu, Y., Connelly, K., Paul, G., Dick, A., Wright, G., 2009. Evaluation framework for algorithms segmenting short axis cardiac MRI. MIDAS J. - Card. MR Left Ventricle Segmentation Challenge.

Schaerer, J., Casta, C., Pousin, J., Clarysse, P., 2010. A dynamic elastic model for segmentation and tracking of the heart in MR image sequences. Med. Image Anal. 14, 738-749.

Shi, Y., Karl, W., 2008. A real-time algorithm for the approximation of level-setbased curve evolution. IEEE Trans. Image Process. 17, 645-656.
Ulén, J., Strandmark, P., Kahl, F., 2013. An efficient optimization framework for multi-region segmentation based on lagrangian duality. IEEE Trans. Med. Imag.. Uzunbas, M., Zhang, S., Pohl, K., Metaxas, D., Axel, L., 2012. Segmentation of myocardium using deformable regions and graph cuts. In: IEEE International Symposium on Biomedical Imaging (ISBI). IEEE, pp. 254-257.

Wijnhout, J., Hendriksen, D., Assen, H., Geest, R., 2009. LV challenge LKEB contribution: fully automated myocardial contour detection. MIDAS J. - Card. MR Left Ventricle Segmentation Challenge, 683.

World Health Organization, 2012. Cardiovascular Diseases. Fact Sheet 317. 